\title{
Choice Between Reinforcers With and Without Delayed Shock Effects of a Pre-Shock Signal
}

\author{
Haleh Amanieh \\ West Virginia University, ha0099@mix.wvu.edu
}

Follow this and additional works at: https://researchrepository.wvu.edu/etd

\section{Recommended Citation}

Amanieh, Haleh, "Choice Between Reinforcers With and Without Delayed Shock Effects of a Pre-Shock Signal" (2021). Graduate Theses, Dissertations, and Problem Reports. 8326.

https://researchrepository.wvu.edu/etd/8326

This Thesis is protected by copyright and/or related rights. It has been brought to you by the The Research Repository @ WVU with permission from the rights-holder(s). You are free to use this Thesis in any way that is permitted by the copyright and related rights legislation that applies to your use. For other uses you must obtain permission from the rights-holder(s) directly, unless additional rights are indicated by a Creative Commons license in the record and/ or on the work itself. This Thesis has been accepted for inclusion in WVU Graduate Theses, Dissertations, and Problem Reports collection by an authorized administrator of The Research Repository @ WVU. For more information, please contact researchrepository@mail.wvu.edu. 
Choice Between Reinforcers With and Without Delayed Shock: Effects of a Pre-Shock Signal

\author{
Haleh Amanieh \\ Thesis Submitted \\ to the Eberly College of Arts and Sciences \\ at West Virginia University \\ in partial fulfillment of the requirements for the degree of \\ Master of Science in \\ Psychology
}

Michael Perone, Ph.D., Chair

Karen Anderson, Ph.D.

Hawley Montgomery-Downs, Ph.D

Department of Psychology

Morgantown, West Virginia

2021

Keywords: choice, signaled punishment, delayed punishment, conflicting consequences Copyright 2021 Haleh Amanieh 


\begin{abstract}
Choice Between Reinforcers With and Without Delayed Shock: Effects of a Pre-Shock Signal

Haleh Amanieh

Choice research has largely emphasized situations in which individuals choose between a smaller-sooner reward and a larger-later reward. However, in many situations, choosing a certain one of the options results in conflicting consequences: an immediate reinforcer and a delayed punisher. Recent research with rats has evaluated how choice between a smaller reinforcer alone and a larger reinforcer with a delayed shock changes as a function of shock intensity and delay. Research in this area has also evaluated the effects of signals that follow the response and precede shock with conflicting results. The present experiment investigated a different signaling procedure, one that only precedes shock, in a choice procedure. Rats chose between one food pellet and two pellets with a delayed shock. Shock intensity and signal presence were manipulated across conditions. Using an adjusting delay procedure, the delay to shock was adjusted based on the rats' choices. Choice for one food pellet raised the delay to shock in subsequent trials and choice for two pellets with shock reduced the delay in subsequent trials. Adjustments continued within each condition until the rat chose both options equally (indifference) or until choice for the two pellets with shock persisted. In general, higher shock intensities devalued the two pellets to the greatest extent, resulting in longer adjusting delays before indifference was reached. The signal, however, had no consistent effect on choice. Procedural differences across experiments evaluating signaled shock are discussed as possible explanations for conflicting findings regarding the effect of the signaled shock on behavior.
\end{abstract}




\section{ACKNOWLEDGEMENTS}

Several individuals have contributed to my success as an academic and my growth as a person. Thank you to my mentors at the University of Florida for being so supportive of my interest in behavior analysis, for providing me countless opportunities, and for modeling genuine caring mentorship: Dr. Nicole Dorey, Dr. Jonathan Fernand, Dr. Jennifer Haddock, among several others. Thank you to my committee members for providing their brilliant insights to this project. Thank you to Dr. Anderson and Dr. Montgomery-Downs for being inspirational and endlessly supportive. Thank you to Dr. Perone for teaching me more than I wished to know about aversive control and for sharing his clever data analytic techniques. My sanity during the thesis process was kept together by my wonderful support system: Jarod Roberts, Morissa Edery, Bri Sarno, Dayla Casey, Kayci Anderson, and of course my mother. Ya'll inspire me and keep me together every day and I'm grateful to have had you through this process and beyond. 


\section{Table of Contents}

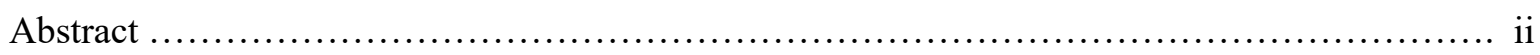

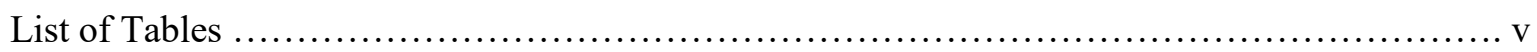

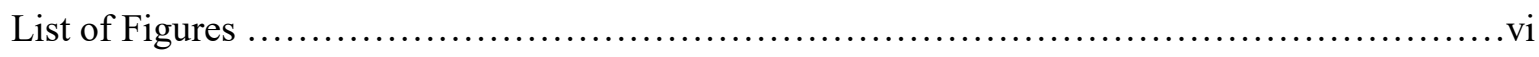

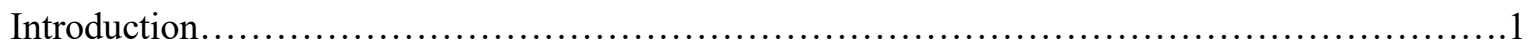

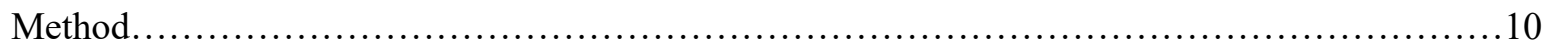

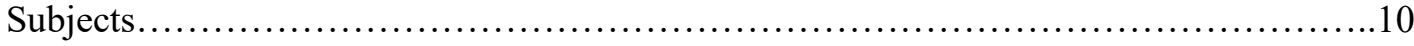

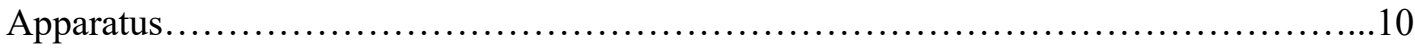

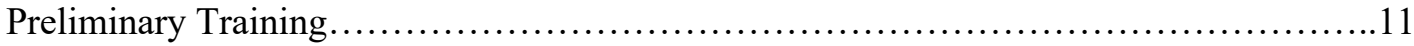

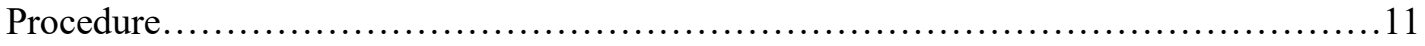

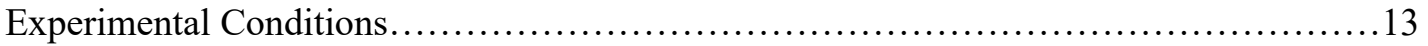

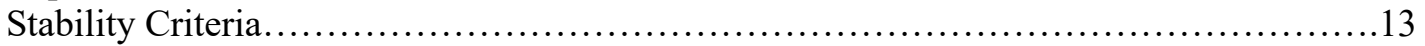

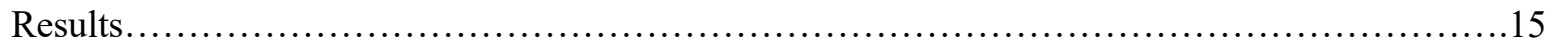

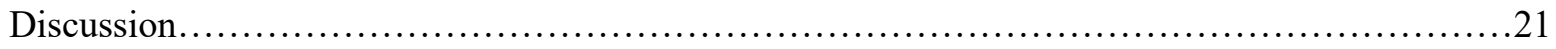

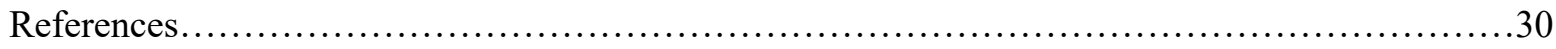




\section{List of Tables}

Table 1. Experimental conditions for each rat (SR1, SR2, SR25, SR4) and the number of sessions in each. The conditions are defined in terms of the intensity of the shock $(\mathrm{mA})$ and the presence or absence of a signal preceding shock. Conditions are listed in order of presentation. Except in the last two conditions of Rat SR2, shocks lasted 200 ms..............................................

Table 2a. No-Signal Conditions: Stable adjusting delays (s), forced-choice latencies (s) $\left(25^{\text {th }}, 50^{\text {th }}\right.$, $75^{\text {th }}$ percentiles) and results of sign tests based on the 96 stable blocks of each condition. A '+' was assigned when a dual-valence latency was shorter than a single-valence latency, and a '-' was assigned when a dual-valence latency was longer than a single-valence latency. A difference of 0 was not included in the analysis. The right most column shows the p-values that correspond with the sign tests. Missing $p$-values indicate that the results were not statistically significant...36

Table $2 b$. Signal Conditions: Stable adjusting delays (s), forced-choice latencies (s) $\left(25^{\text {th }}, 50^{\text {th }}\right.$, $75^{\text {th }}$ percentiles) and results of sign tests. Details as in Table 2a

Table 3. The number of Signal and No-Signal conditions in which the sign test results showed that significantly less or more than 50 percent of shorter latencies occurred in the dual-valence trial, or with no significant difference. Also shown is the proportion of conditions in which a greater number of shorter latencies occurred in the dual-valence trial. 


\section{List of Figures}

Figure 1. Stable adjusting delays to shock for Rat AD10 at three shock intensities with and without a signal. Redrawn from Dumas (2014).

Figure 2. Mean response latencies of groups of college students at several delays to money loss with and without a signal. The dashed line shows the mean response latency during the unpunished baseline condition. Redrawn from Trenholme and Baron (1975).

Figure 3. Rat SR25's adjusting delay across blocks in the condition in which the last 30 sessions (480 blocks) were used to calculate the indifference point. The arrow designates the block at which stable sessions began

Figure 4. Stable adjusting delays to shock from all Signal and No-Signal conditions as a function of shock intensity. Bars are displayed in order of presentation within each shock intensity. Shocks lasted $200 \mathrm{~ms}$ except in Rat SR2's last two conditions at $0.8 \mathrm{~mA}$. Here, the shocks lasted $300 \mathrm{~ms}$ and $500 \mathrm{~ms}$, respectively. Note that the scale on the y-axis differs across rats.

Figure 5. Adjusting delay to shock (s) plotted across the first 200 blocks at the $0.8 \mathrm{~mA}$ and 1.0 $\mathrm{mA}$ shock intensities. Numbers next to data paths indicate the order of conditions within each shock intensity. Breaks in the second data path for Rat SR4 are a result of incomplete sessions. Note that the scale on the $y$-axis differs across rats.

Figure $6 a$. Percentage of trials that the latency to respond was shorter in the dual-valence trial and in the single-valence trial during forced-choice trials in stable sessions of each No-Signal condition. The left column shows results for conditions with adjusting delays at or below $12 \mathrm{~s}$; the right column shows results for conditions with adjusting delays above $12 \mathrm{~s}$. The asterisks above pairs of bars indicate that the difference in number of longer and shorter latencies across the two levers was statistically significant.

Figure $6 b$. Percentage of trials that the latency to respond was shorter in the dual-valence trial (shaded) and in the single-valence trial (white) during forced-choice trials in stable of each Signal condition across shock intensity. Details as in Figure 6a....

Figure 7. Percentage of trials with a shorter latency to press the dual-valence lever during the stable sessions of Signal and No Signal conditions 


\section{Choice Between Reinforcers With and Without Delayed Shock: Effects of a Pre-Shock}

\section{Signal}

Skinner's (1953, pp. 230-241) analysis of self-control was concerned with situations in which a single instance of behavior produces conflicting consequences. For example, drinking large amounts of alcohol results in social reinforcers in the short term, but may be followed by a painful hangover the next day. A person with lactose intolerance might consume dairy-rich foods that are delicious, but later cause gastrointestinal discomfort.

Most behavior-analytic research on self-control does not address Skinner's (1953) analysis. Instead, research on self-control is usually conducted with a delay of gratification paradigm (Madden \& Bickel, 2010). The paradigm involves choice between a large reinforcer available after a delay and a small reinforcer available immediately. Only a few studies have been conducted to investigate Skinner's conceptualization of self-control using a choice paradigm with conflicting consequences. These studies are reviewed below along with studies that evaluate the impact of signaling delayed consequences, which has direct relevance to the present experiment.

One approach to studying the type of self-control described by Skinner (1953) involves providing a choice between a small reinforcer alone and a larger reinforcer with an aversive outcome that occurs after a fixed delay. For example, in a study by Rodriguez et al. (2018), rats chose between one food pellet (a "single-valence" consequence) and four food pellets followed by a delayed shock (“dual-valence” consequence). The delay to shock was manipulated within each session from $0 \mathrm{~s}$ to $40 \mathrm{~s}$. Rats chose one pellet almost exclusively when four pellets were followed by an immediate ( 0 -s delay) shock. As delay was raised, the rats chose the dual-valence consequence progressively more often and chose it almost exclusively when shock was delayed 
by $40 \mathrm{~s}$. Rodriguez et al. also found that raising the delay to shock decreased the latency to press the dual-valence lever, whereas the latency to press the single-valence lever was unaffected. Liley et al. (2019) used a similar procedure to evaluate differences in female and male rats' choices between one pellet alone and three pellets with a delayed shock $(0,4,8,12$ or $16 \mathrm{~s})$. They found that female rats were more sensitive to the shock across delays, but that both sexes chose the three pellets progressively more often as the delay to shock was raised. These experiments show that raising the delay to shock reduces the effect of the shock on choice. Immediate, reinforcing consequences have more control over responding when aversive consequences are relatively remote.

In another study involving consequences with both appetitive and aversive outcomes, Woolverton et al. (2012) evaluated the effects of delayed punishment on the self-administration of cocaine with monkeys. Monkeys chose between two options each resulting in cocaine followed by histamine, but the histamine injection occurred immediately for one option and after various delays ( 1 to $720 \mathrm{~s}$ ) for the other option. Histamine has been found to function as a punisher when made contingent on a response (Woolverton, 2003). Histamine and cocaine doses were the same across the options, and delays were manipulated across conditions. When the delays were short, the monkeys were biased towards one of the two levers such that the preferred lever was chosen in more than 85 percent of trials. Delay to histamine was increased for the consequence associated with the non-preferred lever and choice for that consequence increased as a function of the delay. Woolverton et al.'s findings demonstrate that when both alternatives result in the same magnitude reinforcer and punisher, the delay to punishment has a substantial effect on choice. Their findings also demonstrated that delay reduces the effects of the punisher to the extent that it can overcome a side bias. 
To further analyze choice in situations with conflicting consequences, Dumas $(2014)^{1}$ used a procedure to quantify the degree to which a delayed shock degraded the reinforcing value of two pellets to equal that of one pellet. Pressing a single-valence lever produced one pellet and pressing a dual-valence lever produced two pellets and a delayed shock. The intensity and duration of the shock were manipulated across conditions. The delay was adjusted within each condition based on each rats' choices using a procedure developed by Mazur (1987).

The adjusting-delay procedure involved sessions consisting of 16 blocks of four trials. The first two trials were forced-choice trials: Just one of the levers was inserted into the chamber per trial, ensuring that the rat contacted the consequences associated with each lever. The rat was then exposed to two free-choice trials in which it could press either lever. The rat's choices during free-choice trials led to adjustments in the delay to shock in the next block of trials. If the rat chose the single-valence consequence in both trials, the delay was raised by $2 \mathrm{~s}$ in the next block. If the rat chose the dual-valence consequence in both trials, the delay was reduced by $2 \mathrm{~s}$. If the rat chose each consequence once, the delay remained unchanged. Adjustments in the delay to the shock continued until the rat demonstrated indifference, that is, until it consistently split its free choices between the two types of consequences. At indifference, the delayed shock degraded the value of the two food pellets in the dual-valence consequence such that it was equal to the value of the one pellet in the single-valence consequence.

Figure 1 shows some of Dumas's (2014) results. This graph shows the adjusting delay to shock as a function of shock intensity for a representative rat. Increasing the shock intensity reduced the value of two pellets. When shocks were highly effective, rats continued to choose the single-valence consequence until the adjusting delay to shock was sufficiently long to reduce

\footnotetext{
${ }^{1}$ An unpublished thesis and follow-up work, hereby referred to as Dumas (2014).
} 
the effects of the shock. Adjusting delays were progressively longer as the shock intensity was raised, resulting in the increasing function shown in Figure 1. Therefore, the adjusting delay is directly related to the rat's sensitivity to shock. The method used in Dumas's experiment provides a measure for assessing how the parameters (i.e., the intensity) of delayed shock affect choice.

To identify variables that might increase the effectiveness of delayed shock, Dumas (2014) added a signal during the delay. In conditions with the signal, a press on the dual-valence lever resulted in a flashing houselight for the duration of the delay to shock. In conditions without the signal, sessions took place as described above. Figure 1 shows results for a representative rat. The signal did not increase the effectiveness of shock.

Toegel (2018; Experiment 2) studied a different signaling procedure within the conflicting-consequence paradigm. His procedure was based on Trenholme and Baron's (1975) study of delay of punishment. College students pressed a button that randomly $(p=.5)$ produced either money gain immediately or money loss (the punisher) after a delay that differed across groups from $0 \mathrm{~s}$ to $40 \mathrm{~s}$. For some groups, white noise was presented for $1 \mathrm{~s}$ immediately after a response and again $1 \mathrm{~s}$ before the onset of money loss. Figure 2 shows the mean latency to respond by each group relative to an unpunished baseline (i.e., without any money loss). Response latencies were longest - responding was weakest - in the group with immediate punishment (0-s delay) and were progressively less affected as the delay to punishment was raised. In the group with punishment delayed by $40 \mathrm{~s}$, latencies were similar to the group with no punishment. The addition of the signal increased the effectiveness of the delayed punishment for all groups. When punishment was delayed by $40 \mathrm{~s}$, which previously had little effect on 
responding, the signal resulted in latencies that were nearly three times longer than when the signal was absent.

Toegel (2018) adapted this signaling procedure for his experiment with rats. His signal consisted of a flashing houselight and pulsing white noise for $2 \mathrm{~s}$ immediately after food delivery and again for $2 \mathrm{~s}$ before the onset of shock. Despite the use of a signaling procedure that was successful in Trenholme and Baron's (1975) study, there was no discernable effect.

As in Dumas's (2014) research, Liley et al. (2019) tested the effects of a signal spanning the delay from the lever-press response to the shock. Rats chose between one pellet alone and three pellets with a delayed shock. The delays $(0,2,4,8,16 \mathrm{~s})$ were manipulated within each session. The signal was the illumination of a cue light located above the dual-valence lever. In conditions without the signal, choice for the dual-valence consequence increased as the delay to shock was raised. When the signal was added, choice for the dual-valence consequence was significantly reduced. In contrast to Dumas' (2019) results, the presence of the signal throughout the delay increased the effectiveness of the aversive consequence.

MacDonald (1973) used a different method to evaluate the effects of a signal spanning the delay between a response and a shock. Rats pressed a lever during a two-component multiple schedule. Each component, labeled S1 and S3, was differentiated by a different stimulus. In addition, each component consisted of a two-link chain schedule. Pressing the lever in the presence of the first link in the chain (either S1 or S3) resulted in the presentation of the second link in the chain on a variable-interval (VI) 90-s schedule. Completing the schedule during S1 resulted in a stimulus change indicating that the second link of the chain (S2) was in effect. Similarly, completing the schedule during S3 resulted in a stimulus change indicating that the second link of the chain (S4) was in effect. During the second link in both components, a 
response on the lever produced a dipper with evaporated milk for $2 \mathrm{~s}$ after an $8 \mathrm{~s}$ delay. The second link was in effect until 20 reinforcers were delivered or until 20 min elapsed. For each link in the chain, a different stimulus was present to make each link discriminable from one another. In the baseline phase, response rates during all four links were recorded to establish baseline rates of responding. In a subsequent phase, the same schedule of reinforcement during the second link was in effect as in baseline. However, shocks were made contingent on responding during the second link on a variable-ratio (VR) 10 schedule. In S2, a buzzer (the signal) turned on following the punished response for $6 \mathrm{~s}$. The offset of the buzzer was followed immediately by a shock. In S4, a shock followed the punished response after a 6 s delay with no signal spanning the delay. In general, responding was higher in S1 and S2 relative to S3 and S4, respectively, suggesting that the signal did not increase the effectiveness of the delayed punisher. The effects of the signal that spanned the delay between the punished response and the subsequent shock were opposite of that found by Liley et al. (2019).

Dumas (2014) and Toegel (2018) tested two signaling procedures in an effort to increase the effectiveness of delayed shock in the conflicting-consequence paradigm. Neither were successful. In contrast, Liley et al. (2019) found that a signal similar to the one used by Dumas increased the effectiveness of delayed shock and MacDonald (1973) found that the signal spanning the delay decreased the effectiveness of delayed shock. These conflicting results necessitate more research in this new area before the role of signals can be understood. Exploring other methods to signal the delayed shock would allow for a more comprehensive understanding of factors that influence choice in the conflicting-consequence paradigm. The present experiment examined a signaling procedure studied by Badia and colleagues in various experiments, outlined below. 
Badia and colleagues evaluated the effects of a signal when shocks were delivered independent of responding. For example, Badia and Culbertson (1972; Experiment 2) examined rats' preferences between signaled and unsignaled shock by allowing rats to press a lever to produce a condition in which inescapable shocks were signaled. Each session began with an unsignaled shock condition in which shocks were delivered after varying periods of time, regardless of the rat's behavior. A lever press produced a 3-min period in which a tone preceded each shock by $5 \mathrm{~s}$. After the 3-min period elapsed, the unsignaled shock condition resumed until the lever was pressed again. All rats remained in the signaled condition from about 80 to 95 percent of session time.

The finding that rats prefer signaled over unsignaled response-independent shocks has been replicated across a variety of shock intensities. Badia, Culbertson, and Harsh (1973; Experiment 2) compared signaled and unsignaled shocks when the intensity of the signaled shock was raised while the unsignaled shock remained the same. At the start of the experiment, both shock intensities were $1.0 \mathrm{~mA}$. The intensity of the signaled shock was raised in either 0.4or $0.2-\mathrm{mA}$ steps across conditions. Rats continued to prefer the signaled shock even though the intensity of the shock in the signaled situation was higher than that of unsignaled shocks. When the intensity of signaled shock was $2.2 \mathrm{~mA}$, rats spent an average of 65 percent of sessions in the signaled shock conditions even though the intensity of the unsignaled shock was less than half at $1.0 \mathrm{~mA}$.

The preference for signaled shock also was found when signaled and unsignaled shocks differed in frequency. Badia, Coker, and Harsh (1973) allowed rats to choose to remain in a condition which signaled shocks occurred at a high rate or to change conditions in which unsignaled shocks were delivered at a lower rate. Rats preferred signaled shocks even when they 
occurred twice as often as unsignaled shocks. The literature regarding signaled, responseindependent shock suggests that a signal preceding shock can successfully reduce the effects of delayed shock.

The majority of research evaluating the effects of a signal preceding shock has been evaluated using a preference measure as described above. Other researchers have measured the degree to which responding is suppressed when response-independent shocks are signaled compared to when they are not signaled. Imada (1972) measured water-deprived rats' licking behavior during 5-min sessions in which rats were given free access to water. In the first phase (baseline), no shocks were delivered. Then, five unsignaled shocks were delivered in irregular intervals across the 5-min session. In the last phase, a 5-s tone preceded each shock. Licking was most suppressed when shocks were unsignaled. When the signal was introduced, licking behavior was recovered to near-baseline levels for most rats. Similar findings on the effects of the signal on response suppression were observed in various experiments measuring licking (Miyashita, 1971; Nageishi \& Imada, 1974) and lever pressing maintained by positive reinforcement (Davis \& McIntire, 1969; Weiss \& Strongman, 1969; for a review, see Badia et al., 1979).

Another approach to studying the degrees of response suppression by signaled and unsignaled response-independent shock was conducted by MacDonald and Baron (1973). The method used in their study was similar to the two-component multiple schedule used by MacDonald (1973) described above. However, the events that occurred during the second link in the chain (S2 or S4) differed. The second links were in effect for 5 min. During baseline, a dipper consisting of evaporated milk was presented response-independently once every $15 \mathrm{~s}$, on average, in S2 and S4. In general, response rates during the S1 and S3 were undifferentiated 
during baseline. In a subsequent phase, shocks were delivered during S2 and S4 responseindependently once every 4 min, on average. However, shocks were preceded by a 5 -s buzzer in $\mathrm{S} 4$, but not S2. Response rates during the first link in the chain was lower in S1 relative to S3, suggesting that the unsignaled shock had a greater suppressive effect than the signaled shock. When the component in which the signal was present was switched, that is when the buzzer preceded shock in S2, but not S4, response rates were again suppressed to a greater extent in the first link of the chain associated with unsignaled shock. Taken together, findings from experiments evaluating response suppression using various methods provide strong evidence that signals preceding shock reduce the suppressive effects of the shock.

Fewer studies have evaluated the effects of a signal preceding shock when shocks were delivered response-dependently (for a review, see Hymowitz, 1979). For example, Hymowitz (1973a; Experiment 3) trained rats to press a lever for food on a VI-35 s schedule. After measuring baseline response rates, rats were divided into groups in which a fixed-interval (FI) 65-s or VI-65 s shock schedule was superimposed on the VI-35 s food schedule. Groups were further divided into conditions in which the probability of a 5-s signal (the illumination of three indicator lights) preceding each shock was $0 \%, 50 \%$, or $100 \%$. Regardless of the shock schedule, the least amount of response suppression occurred when shocks were always preceded by a signal. Overall, studies evaluating the effects of signaled shock by arranging responseindependent or response-dependent shock were consistent in finding that the signal reduces the aversiveness of the shock across a variety of dependent measures.

The following experiment is essentially a replication of Dumas's (2014) study with Badia's signaling procedure. The purpose was to test a signaling procedure within the conflicting-consequence paradigm to find out if any change in sensitivity to shock can be 
produced. Rats chose between one pellet or two pellets and a delayed shock. In some conditions, a 5-s signal preceded the shock. Effects of a signal on sensitivity to shock were evaluated by comparing the stable adjusting delay across conditions differing in shock intensity and signal presence. In contrast to the previous work on conflicting consequences where the purpose of the signal was to increase the effect of shock, the signal in the proposed work is expected to reduce the effect.

\section{Method}

\section{Subjects}

Four male Sprague Dawley rats were maintained at 80 percent $( \pm 2 \%)$ of their freefeeding body weights by food reinforcers delivered during the experimental sessions and supplemental feedings of standard lab chow in the home cage at least 30 min after the sessions. Target weights were adjusted periodically according to a growth chart for Sprague Dawley rats. The rats were housed in pairs in a temperature-controlled room with a 12:12 hr reversed light/dark cycle and water was freely available in the home cages. The rats had been in an experiment concerned with extinction and spontaneous recovery after training on variableinterval schedules of food reinforcement.

\section{Apparatus}

Sessions were conducted in four operant-conditioning chambers enclosed in ventilated sound-attenuating chests (Med Associates Inc., St. Albans, VT). The interior of each chamber was $29 \mathrm{~cm}$ long, $22 \mathrm{~cm}$ high, and $24 \mathrm{~cm}$ deep. The ceiling and sidewalls were constructed of Plexiglas and the end walls of stainless steel. The floor consisted of 19 stainless-steel rods $0.5 \mathrm{~cm}$ in diameter, spaced approximately $1.3 \mathrm{~cm}$ apart. On the front wall were two retractable levers. Each lever was $4.4 \mathrm{~cm}$ wide, $1.3 \mathrm{~cm}$ thick, and protruded $1.9 \mathrm{~cm}$ into the chamber when inserted. 
The inside edges of the levers were spaced $11.4 \mathrm{~cm}$ apart $(5.7 \mathrm{~cm}$ from the middle of the wall). The tops of the levers were positioned $8 \mathrm{~cm}$ from the floor. White cue lights (No. $1820 \mathrm{bulb}$ ) were located approximately $5 \mathrm{~cm}$ above each lever. An audio speaker was located behind the back wall. General illumination was provided by a houselight (No. 1820 bulb) located on the back wall. White noise $(80 \mathrm{~dB})$ masked extraneous sounds. Food pellets (45-mg, BioServ) were delivered into a feeder centered on the front wall. Each pellet was accompanied by a $1000-\mathrm{Hz}$ tone lasting $1 \mathrm{~s}$. When multiple pellets were delivered, the accompanying tone was extended by 1 s per pellet. The levers were retracted for the duration of the tone. Aversive stimulation consisted of a scrambled foot shock from a constant-current shock generator (Med Associates Model ENV-413). Experimental events were controlled and recorded with computers connected to the chambers via digital interfaces (Measurement Computing Model PCI-PDIS08) running programs written in Visual Basic.

\section{Preliminary Training}

Because the rats' prior experience involved responding on a single lever, preliminary training was conducted to engender responding on both levers. Training began with the insertion of one lever. Each press produced a food pellet. After 10 pellets were delivered, the lever was retracted and the other lever was inserted, and again each press produced a pellet. The levers were alternated in this way until 100 pellets were delivered. Three to five sessions were conducted for each rat and each session lasted approximately 10 minutes.

\section{Procedure}

Sessions were normally conducted seven days per week at approximately the same time each day. A 5-min blackout period preceded the start of each session during which the chamber was dark and silent, and the levers were retracted. This allowed the rat to recover from handling 
and transportation between the vivarium and the laboratory. After the blackout, the houselight and white noise were turned on and the session began.

Each session consisted of blocks of four trials: two forced-choice trials followed by two free-choice trials. Sessions lasted for 16 blocks or $300 \mathrm{~min}$, whichever occurred first.

The two forced-choice trials at the beginning of each block ensured that the rat contacted the programmed consequences on each lever. A single lever was inserted into the chamber and presses produced the consequences corresponding to that lever: one pellet by pressing the singlevalence lever or two pellets and a delayed shock by pressing the dual-valence lever. The first forced-choice trial in the first block of a session involved the dual-valence lever. This was in order to yoke the trial duration to trials with the single-valence lever, if necessary; the yoking procedure is described below. With the exception of the first block, the lever in the first forcedchoice trial was selected at random in every odd-numbered block. Even-numbered blocks began with the lever that had been used in the second trial of the previous block.

Each free-choice trial began with both levers inserted into the chamber. After a leverpress response, the levers were retracted and the consequence corresponding to the pressed lever was delivered. As in Mazur's (1987) procedure, the rat's response allocation during the freechoice trials determined the delay to shock in the subsequent block of trials. If the dual-valence lever was pressed in both trials, the delay was raised by $2 \mathrm{~s}$. If the single-valence lever was pressed in both trials, the delay was lowered by $2 \mathrm{~s}$. If each lever was pressed once, the delay remained unchanged. At no point was the delay to shock lowered to less than $10 \mathrm{~s}$. This was to prevent consumption of food pellets to be followed closely by a shock.

Trials were programmed every $90 \mathrm{~s}$ with the constraint that a minimum of $15 \mathrm{~s}$ separated the end of a consequence (one pellet after pressing the single-valence lever or the delayed shock 
after pressing the dual-valence lever) from the start of the next trial. This prevented temporal contiguity between the consequence and the onset of the next trial. If the delay to shock became sufficiently long to extend a trial duration beyond $90 \mathrm{~s}$, a yoking procedure prevented trial durations from differing between the two types of trials. If a dual-valence trial lasted longer than $90 \mathrm{~s}$, the duration of the following single-valence trial was yoked to the duration of the dualvalence trial. A new yoked trial duration was established with each block's first forced-choice dual-valence trial.

\section{Experimental Conditions}

Table 1 shows the sequence of experimental conditions for each rat. The conditions differed primarily in terms of shock intensity and the presence or absence of a signal. In conditions with the signal, the shocks were preceded for $5 \mathrm{~s}$ by a $1500-\mathrm{Hz}$ tone. The rats were first exposed to shock intensities in ascending order; Rats SR1 and SR2 received unsignaled shocks in these conditions and Rats SR25 and SR4 received signaled shocks. The rats that had received signaled shock then received several conditions with unsignaled shocks and vice versa. After this initial sequence, the subsequent conditions differed depending on individual results. Selected conditions were replicated to clarify the nature of the results. The shocks lasted $200 \mathrm{~ms}$, except in Rat SR2's last two conditions, in which the duration was raised to $300 \mathrm{~ms}$ and $500 \mathrm{~ms}$.

\section{Stability Criteria}

Each condition lasted until response allocation in the free-choice trials over six consecutive sessions was stable. Stable responding was characterized in two different ways. When responding was allocated evenly between the single-valence and dual-valence levers, it was judged as indifferent to the consequences associated with the two levers. To identify stable indifference, each of the last six sessions was divided into half-sessions consisting of eight 
blocks. The mean adjusting delay was calculated for each half-session. Choice was considered indifferent when the following criteria were satisfied:

1. All trials must have been completed in every session.

2. The proportion of free-choice responses on either lever could not form a monotonic trend.

3. Neither the highest nor lowest half-session mean delay occurred within the last six halfsessions.

4. The mean delay over the last six half-sessions was neither the highest nor the lowest of the condition.

5. The mean delay over the last six half-sessions did not differ from the mean of the preceding six half-sessions by more than 10 percent or by more than $1 \mathrm{~s}$ (whichever was larger).

6. For at least five of the six whole sessions, choice of either lever could not occur in fewer than 10 trials or more than 22 trials of the 32 free-choice trials per session. This range was chosen based on probabilities obtained from the binomial distribution. Across 32 choices, if responding were truly indifferent - that is, if the probability of responding on either lever was .5 - the probability of either alternative being chosen less than 10 times or more than 22 times is .01 . This probability was seen as sufficiently extreme to reject the null hypothesis of indifference.

In some conditions, stable responding was not indifferent to its consequences. Instead, the rat always or almost always pressed the dual-valence lever. In this case, a stable adjusting delay was reached in the range of 10 to $12 \mathrm{~s}$ because of the floor effect created by the minimum possible delay of $10 \mathrm{~s}$. A stable adjusting delay in this range was identified by visual inspection of the block-by-block graphs. 
When response allocation was stable, either by reaching indifference or by consistent choice of the dual-valence consequence, the stable adjusting delay was calculated as the mean delay over the last six whole sessions.

These stability criteria were used for all rats in all conditions with the exception of the fifth condition for Rat SR25. For this condition, the stable adjusting delay was calculated across the last 30 sessions (blocks 658 through 1137). Figure 3 shows adjusting delays across blocks for Rat SR25. Responding was cyclical and unlikely to satisfy the stability criteria within a reasonable amount of time.

\section{Results}

The main dependent variable is the mean adjusting delay to shock over the six stable sessions of each condition. At indifference, the reinforcing value of the consequences associated with the dual-valence lever (two pellets and the delayed shock) was equal to the value of the consequences associated with the single-valence lever (one pellet). Indifference is represented by stable adjusting delays above $12 \mathrm{~s}$. However, when the adjusting delay stabilized below $12 \mathrm{~s}$, the value of the two pellets and the delayed shock was higher than that of the one pellet. In this case, the adjusting delay does not represent indifference between the two consequences. Therefore, depending on its duration, the adjusting delay can be conceptualized in two ways: as indifference (when longer than $12 \mathrm{~s}$ ) or as a preference for the dual-valence consequence (when $12 \mathrm{~s}$ or shorter).

Figure 4 shows the final adjusting delay to shock as a function of shock intensity for each rat. Tables $2 \mathrm{a}$ and $2 \mathrm{~b}$ show the adjusting delays with numerical accuracy. In Figure 4, the Signal and No-Signal conditions are indicated by black and white bars, respectively. Bars are shown in order of presentation within each shock intensity. The order of all conditions can be seen in Table 1. In general, adjusting delays were short at lower shock intensities and tended to be longer 
at higher shock intensities. In most conditions with shock intensities of $0.4 \mathrm{~mA}$ or $0.6 \mathrm{~mA}$, choice was almost exclusively for the dual-valence consequence, resulting in shock delays adjusting downward toward the minimum of $10 \mathrm{~s}$. In fact, the adjusting delay stabilized between $10 \mathrm{~s}$ and $12 \mathrm{~s}$ for all rats in their initial exposures to these shock intensities. The shock at these weaker intensities may have reduced the value of the two pellets, but not to the value of one pellet. In the second and third 0.6 mA conditions for Rat SR 4, however, the shock degraded the two-pellet consequence such that choice was indifferent between two pellets with delayed shock versus one pellet.

Higher shock intensities tended to devalue the two pellets. In most conditions with shock intensities of $0.8 \mathrm{~mA}, 0.9 \mathrm{~mA}$, and $1.0 \mathrm{~mA}$, longer delays to shock were required to obtain indifference. However, results for Rat SR2 differed from those of the other rats. Although the first exposure to the $0.8 \mathrm{~mA}$ shock intensity produced a stable adjusting delay that was much longer than in any other condition, the adjusting delays were lower across replications of the condition. Raising the duration of the shock to $300 \mathrm{~ms}$ and then to $500 \mathrm{~ms}$ did not have any effect on choice. During these conditions, stable adjusting delays remained low $(10.1-12.4 \mathrm{~s})$. Rat SR2 did not show sensitivity to either the intensity or duration of the shock in the replications of the $0.8 \mathrm{~mA}$ and $1.0 \mathrm{~mA}$ conditions.

Figure 4 and Tables $2 \mathrm{a}$ and $2 \mathrm{~b}$ also allow comparison of the adjusting delay across the Signal and No-Signal conditions. Because Badia's research indicated that signaling shock makes it less aversive, the signal conditions in the present experiment could be expected to result in lower adjusting delays. To the contrary, the signal did not consistently affect adjusting delays across rats. At any given intensity, there is no consistent difference in adjusting delays in Signal and No-Signal conditions for Rats SR1 and SR2. For Rat SR25, the adjusting delay was shorter 
with the signal in the $1.0 \mathrm{~mA}$ condition. For Rat SR 4, the adjusting delay was longer with the signal in almost every case.

Although no effect of the signal was found in the terminal sessions of each condition, it is possible that the signal may have affected initial adjustments to the shock. Figure 5 shows adjusting delays across the first 200 blocks for the $0.8 \mathrm{~mA}$ and $1.0 \mathrm{~mA}$ conditions for each rat. These shock intensities were selected because all rats were exposed to the intensities in both Signal and No-Signal conditions. The first 200 blocks of a condition represent choice across 12.5 sessions, which was chosen because it was believed to be an adequate sample of behavior at the start of each condition. Adjusting delays initially increased for 7 of 11 (64\%) Signal conditions and for 5 of $12(42 \%)$ No-Signal conditions. Given these comparisons, there is not enough evidence to conclude that the signal reduced the aversiveness of the shock at the start of the condition.

To explore the reinforcing value of the single- and dual-valence consequences further, and how the signal might have affected the reinforcing value of the dual-valence consequence, response latencies were examined. Consequences with relatively high reinforcing value generally produce relatively short latencies whereas consequences with relatively low value produce relatively long latencies. In the present experiment, adjusting delays longer than $12 \mathrm{~s}$ are understood to indicate that the single- and dual-valence consequences have equal value. If this is the case, latency to press the single- and dual-valence levers should not differ systematically. By comparison, adjusting delays of $12 \mathrm{~s}$ or less result from preference for the dual-valence consequence. In such conditions, latency to press on the dual-valence lever should be shorter than the latency to press the single-valence lever. 
To analyze differences in latency, sign tests were conducted on pairs of forced-choice trials in each of the 96 stable blocks of each condition (see Siegel, 1956 for a description of sign tests). Forced-choice trials were selected for this analysis to ensure that each block would include one single-valence response and one dual-valence response. Differences across each pair of latencies were obtained by subtracting the dual-valence latency from the single-valence latency. A positive difference indicates a shorter latency to press the dual-valence lever, suggesting that the two pellets and delayed shock had greater value than the one pellet obtained by pressing the single-valence lever. A negative difference indicates shorter latency to press the forced-singlevalence lever. Differences of zero were rare (across all rats and condition, there were just 24 cases in 4,032 trials $-0.6 \%$ ) and were not included in the analysis. A statistically significant sign test indicates that the disparity between the number of positive and negative latency differences is greater than would be expected by chance if latencies were unaffected by the differences in the single- and dual-valence consequences.

Tables $2 \mathrm{a}$ and $2 \mathrm{~b}$ show the forced-choice latencies and the results of sign tests. A visual representation of the proportion of shorter latencies across the two levers are shown in Figures $6 \mathrm{a}$ and $6 \mathrm{~b}$. The conditions with statistically significant sign tests are marked with an asterisk. The left panel in each figure shows results for conditions in which the adjusting delay can be understood as a preference for the dual-valence lever and the right panel shows results for conditions that ended in indifference between the single- and dual-valence consequences. Low probabilities indicate that one trial type, either the dual- or single-valence trial, consistently produced shorter latencies. Therefore, low probabilities are not consistent with the notion of indifference. Probabilities greater than .05 (represented by a dashed line in the tables) are consistent with the notion of indifference. 
In 13 conditions, the stable adjusting delay was between $10 \mathrm{~s}$ and $12 \mathrm{~s}$ because choice favored the dual-valence consequence. The sign test indicates that dual-valence latencies were consistently shorter in 11 of those conditions. Averaging across the median latencies in these 11 conditions, the dual-valence mean $(0.83 \mathrm{~s})$ was 33 percent lower than the single-valence mean $(1.25$ s) (range: $10 \%-53 \%)$. In Figures $6 a$ and $6 \mathrm{~b}$, the gray bars are larger in majority of these conditions, which indicates that the proportion of shorter latencies in the stable sessions of these conditions was greater in the dual-valence trials relative to the single-valence trials. Generally, both the adjusting delays and latencies indicate that the reinforcing value of the dual-valence consequence was higher than that of the single-valence consequence in these conditions. Rat SR1 is the exception. In the No-Signal condition at $0.4 \mathrm{~mA}$, the non-significant sign test indicates that latencies were not consistently shorter on either lever. In the No-Signal condition at $0.6 \mathrm{~mA}$, the statistically significant sign test indicates that the single-valence latencies were consistently shorter than the dual-valence latencies. The results for these two conditions for Rat SR1 are at odds with the adjusting delay results which indicated that the rat's preference for the dualvalence consequence in these conditions was virtually exclusive.

In 25 conditions, the stable adjusting delays were longer than $12 \mathrm{~s}$ and met the criteria for indifference. The sign test was statistically significant in 15 of these conditions. Averaging across the median latencies in these 15 conditions, the dual-valence mean (1.06 s) and singlevalence mean $(1.03 \mathrm{~s})$ were almost identical. In 7 of the 15 conditions, the single-valence latencies were consistently shorter and in eight conditions the dual-valence latencies were consistently shorter. In the remaining 10 conditions, the non-significant sign tests indicate that latencies were not consistently shorter on either lever. Averaging across the median latencies in these 10 conditions, the dual-valence mean (1.04 s) and single-valence mean (0.98) were within 
about 6 percent of one another. These comparisons are shown visually in the right panels in Figures $6 \mathrm{a}$ and $6 \mathrm{~b}$. Neither the gray nor the white bars are consistently greater across conditions, illustrating the point that neither consequence exerted differential control over latencies. Overall, neither the single-valence nor dual-valence consequence consistently produced shorter latencies, which is compatible with the idea that at steady-state, choice was indifferent between these consequences.

The response latencies can also be used to assess the effects of the signal. If the signal reduced the aversiveness of the shock, one might expect dual-valence latencies to be shorter in the Signal conditions than in the No-Signal conditions. Figure 7 shows the percentage of trials with shorter latencies to press the dual-valence lever in the Signal and No-Signal conditions across shock intensities. For each rat, only shock intensities that had both Signal and No-Signal conditions are shown to allow for comparisons of the signal's effect on latency at each intensity. For Rat SR2, the two conditions that had shock durations of $300 \mathrm{~ms}$ and $500 \mathrm{~ms}$ were not included because those conditions were only conducted without the signal. For ease of comparison, Table 3 shows the number of conditions in which shorter latencies in the dualvalence trial occurred in more than or less than 50 percent of the stable trials as established by statistically significant sign tests, and the number of conditions in which the number of shorter latencies in the dual-valence trials were not consistently different from those in the singlevalence trials, as established by non-significant sign tests. In the No-Signal conditions, shorter latencies favor the single-valence lever in 5 of 14 cases, the dual-valence lever in 4 of 14 cases, and neither lever in 5 of 14 cases. For all rats, the proportion of No-Signal conditions with a greater number of shorter latencies in the dual-valence trials was below 50 percent. This proportion increased when the signal was included. In the Signal conditions, shorter latencies 
favored the single-valence lever in 2 of 15 cases, the dual-valence lever in 8 of 15 cases, and neither lever in 5 of 15 cases. While the proportion of cases with a greater number of shorter latencies in the dual-valence trial increased from the No-Signal condition ( 4 of $14 ; 29 \%)$ to the Signal condition ( 8 of $15 ; 53 \%$ ), the latter proportion is only just above chance. Considering all the results from this analysis provides weak evidence for any effect of the signal.

\section{Discussion}

The present experiment evaluated the effects of a signal preceding shock in the conflicting-consequence paradigm. Rats chose between one pellet or two pellets and a delayed shock. Higher intensity shocks tended to devalue the two pellets to a larger extent than lower intensity shocks. The signal did not have a consistent effect on choice across rats and weak evidence of the signal was found on response latencies. Overall, there was not enough evidence to conclude that the signal had an effect on behavior.

Previous research evaluating choice between a small reinforcer and a large reinforcer with a delayed punisher have found that the parameters of shock affect choice. Dumas (2014) found that higher shock intensities and longer shock durations tend to devalue the larger reinforcer to a greater extent than lower intensity shocks and shorter shock durations. Rodriguez et al. (2018) and Liley et al. (2019) showed that the extent to which the shocks devalue the pellets as part of the dual-valence consequence depends on the delay to shock. The present experiment is consistent with previous research on the differential effects of shock intensity and delay on choice.

The present experiment was primarily concerned with modifying the effects of delayed shock with a signaling procedure. Previous evaluations of signaled shock in choice paradigms have contradicting results. Dumas (2014) and Liley et al. (2019) used a signal that spanned the 
duration of the delay between the delivery of pellets and the onset of shock. Toegel (2018) used a signal that followed the pellets for $2 \mathrm{~s}$ and preceded shock for $2 \mathrm{~s}$. The signal increased the effectiveness of the shock the experiment by Liley et al. but did not systematically affect the punitive efficacy of the shock in experiments by Dumas and Toegel. To further explore the role of the signal in the conflicting-consequence paradigm, the present experiment used a signal that was expected to decrease the aversiveness of the shock. Consistent with Dumas and Toegel, but contrary to Liley et al., no consistent effect of the signal was observed.

One procedural difference between the present experiment and Liley et al. (2019) may account for the difference in findings regarding the effects of the signal. In the present experiment, conditions with the signal were conducted until stability was reached. In contrast, Liley et al. conducted signaled conditions for 10 sessions whereas conditions without a signal were conducted until stability. It may be that there is a transient effect of the signal when it is first introduced that was captured by Liley et al.'s experiment. To identify whether there were any transient effects of the signal on choice in the present experiment, initial adjusting delays were examined. No consistent effect of the signal was found on choice at the start of each condition. The adjusting-delay procedure results in wide fluctuations of adjusting delays throughout a condition before a stable indifference point is reached. It may be that some aspect of choice, such as the maximum or minimum delays reached within a condition, is affected by the signal. Future research should aim to identify whether any aspect of choice is different when the signal is present relative to when it is absent.

Latencies to respond were analyzed to validate the results of the adjusting delays and to see whether there were any differential effects of the signal on latencies. In general, when adjusting delays indicated that the dual-valence had a higher reinforcing value, latencies to press 
the dual-valence lever tended to be shorter. When adjusting delays indicated indifference between the two alternatives, neither consequence consistently produced shorter latencies. The congruency between the two indices of reinforcement value (latencies and adjusting delays) is consistent with the results of latency analyses reported by Dumas (2014) and Rodriguez et al. (2018). In Dumas's experiment, latencies tended to be short in the dual-valence trial when choice favored the dual-valence lever as indicated by the adjusting delays. When adjusting delays indicated indifference, neither lever consistently produced shorter latencies. Rodriguez et al. presented rats a choice between one pellet alone or four pellets with a shock after a fixed delay. Shorter delays to shock resulted in longer latencies to press the dual-valence lever in the forcedchoice trials and choice favored the single-valence consequence. Longer delays to shock resulted in shorter latencies to press the dual-valence lever and choice favored the dual-valence consequence during free-choice trials. Together, results from these studies suggest that the consequences associated with the single- and the dual-valence levers affect choice and latencies similarly.

In contrast to the studies described above, Toegel (2018) found a discrepancy between the latency analysis and choice. In his experiment, rats were given a choice between two pellets after an adjusting delay or two pellets immediately and shock after a fixed delay. Although indifference was reached according to the adjusting delays, latencies to respond favored the dualvalence outcome. Toegel suggested that choice was affected by both the appetitive and aversive consequences, but latencies were under the control of the reinforcing consequence of each lever. Shorter latencies to press the dual-valence lever in his experiment suggests that the immediate delivery of two food pellets was more reinforcing than the delayed delivery of two pellets, despite of the shock following the immediate two pellets. Differences between Toegel's 
procedure and those of the studies described above may account for the contrasting findings. In Toegel's experiment, the dual-valence consequence always resulted in immediate pellets whereas the single-valence consequence resulted in pellets after some delay. In the present experiment, and in experiments by Dumas (2014) and Rodruiguez et al. (2018), pellets were delivered immediately for both options. Future research should evaluate whether different aspects of behavior are differentially affected by reinforcing and punishing events and how those effects are modulated by delays.

The effect of the signal on latencies in the present experiment was equivocal. Similarly, no effect of the signal on latencies were found in Dumas (2014) and Toegel (2018). The signal in Dumas's experiment was a flashing houselight that spanned the entire duration of the delay. Dumas speculated that the flashing houselight may not have been a salient enough signal and the variability of the signal's duration as a result of the adjusting-delay procedure may have accounted for the lack of effect. The signal in Toegel's experiment was a flashing houselight and pulsing white noise that followed a response on the dual-valence lever for $2 \mathrm{~s}$ and preceding shock for $2 \mathrm{~s}$. Toegel suggested that the signal's lack of effect may be because the two iterations of the signal may have opposing effects; the signal following a response may have increased the aversive function of the shock while the signal preceding shock may have reduced the aversive function.

To address these issues, the signal in the present experiment was a tone, similar to that used by Badia and colleagues, and may have been more salient than signals used by Dumas (2014) and Toegel (2018). It was reasoned that the $1500-\mathrm{Hz}$ tone would only be presented before the shock, whereas the white noise and houselight would be present throughout every session of the experiment. The use of a novel stimulus has been found to increase the likelihood of a 
stimulus-stimulus pairing (Pierce \& Cheney, 2013). In addition, the signal in the present experiment occurred in a single temporal location (preceding shock) and was a consistent duration ( $5 \mathrm{~s}$ preceding shock) and was thought it might exert more effect on behavior than the signal used in the previous studies. However, there was no clear effect of the signal on behavior in the present study.

The absence of an effect of the signal in the present experiment and in research by Dumas (2014) and Toegel (2018) may be attributed to the fact that the signal was redundant. Pressing the dual-valence lever and the presentation of two pellets was consistently followed by shock. The addition of the signal was redundant with these two predictive stimuli. It is curious, however, that signals used by Liley et al. (2019) and Trenholme and Baron (1975) had a behavioral effect despite being redundant with other stimuli. In Liley et al., pressing the dualvalence lever and the presentation of multiple pellets always preceded shock. In Trenholme and Baron, a press of the button resulted in an equal probability of either immediate reinforcement or delayed punishment. A press of the button that did not result in reinforcement was perfectly correlated with the delayed presentation of punishment. The addition of the signal during the delay was redundant with the absence of the reinforcing stimulus. The contradictory findings regarding the effect of the signal across these studies warrant further research on the behavioral effect of the redundancy.

To evaluate the behavioral effect of the redundancy, a second experiment was planned that involved the presentation of probabilistic shock. Probabilistic shock as part of the dualvalence consequence would eliminate the predictive relation between shock and pressing the dual-valence lever and the two pellets, thereby eliminating the redundancy of the pre-shock signal. Unfortunately, because of time constraints caused by the global pandemic, the second 
experiment was not completed. Future research might shed light on whether the lack of consistent effect of the signal on choice is due to redundancy modifying the conflictingconsequence procedure to include probabilistic shock.

The signaling procedure used in the present experiment was based on that used by Badia and colleagues. In their experiments, rats were given response-independent shocks and responded to produce signals (5-s tone) that preceded the shocks. Rats consistently responded to produce signaled shock. Signal that precedes shock have been found to reduce response suppression when shocks were presented response-independently (e.g., MacDonald \& Baron, 1973) and response-dependently (e.g., Hymowitz, 1973b). In the present experiment, a signal was arranged similarly (5-s tone preceding shock) and was expected to reduce the aversiveness of the delayed shock, thereby resulting in shorter adjusting delays relative to when the signal was absent. However, there was no consistent effect of the signal. Other than the potential redundancy of the signal discussed above, one major procedural difference may have accounted for the discrepancy in findings. In the present experiment, the response that produced signaled shock also produced two food pellets. There were multiple variables that affected behavior (two pellets, changing delays to shock, the presence of the signal, shock intensity) and therefore, the controlling effect of the signal may have been superseded by the other variables. In contrast, there were fewer variables in the studies listed above, which may have resulted in a more salient effect of the signal. Future research should evaluate the effect of the signal in simpler situations, for example, one with a single response option, to systematically evaluate the interactions between parameters of reinforcers and punishers arranged for that response.

The prevailing theoretical account for the effect of the signal that precedes shock on behavior is the safety signal hypothesis (Badia et al., 1979; Harsh \& Badia, 1975; Hymowitz, 
1979; Perkins et al., 1966; Price \& Geer, 1972; Seligman, 1968). The safety signal hypothesis states that a stimulus that precedes aversive stimuli divide the situation into safe and unsafe periods. When shock is preceded by a signal, safe (shock-free) periods and unsafe (impending shock) periods are discriminable because of the presence of the signal. Although signaled shock situations have been found to be preferred and have been found to result in less response suppression relative to unsignaled shock, it has been found that the signal itself acquires conditioned aversive properties and can be used to punish behavior (McAllister \& McAllister, 1971). To address this seemingly contradictory finding, the safety hypothesis emphasizes that behavior in these situations is largely controlled by safe periods that can only be discriminated when shock is signaled.

The discrepancies in findings regarding the effect, or lack of effect, of the signal across the studies described so far may be accounted for by the safety signal hypothesis. It has been generally found that variables that affect the effectiveness of the signal preceding shock in producing a preference or reducing response suppression include both features of the signal and features of the safety period. For example, Harsh and Badia (1976) manipulated the duration of the safety period while keeping the signal that preceded shock constant. Shocks were delivered response independently, and rats chose between signaled and unsignaled shock. They found that choice for signaled shock varied as a function of the safety period duration. Shorter safety period durations weakened preference for signaled shock while longer safety period durations strengthened it. Therefore, it may be that the method used by the present study compared to other work involving a signal preceding shock resulted in a change in the periods that signaled safety, thereby changing the function of the signal. More specifically, changes in the delay to shock across blocks using the adjusting delay procedure results in changes in the duration of the safety 
period. A careful evaluation or manipulation of features during the safety period in the conflicting-consequence paradigm may shed light on the role of the signal in such choice situations.

Future research should aim to identify methods that modify choice when one alternative results in a dual-valence outcome. Many choices made by humans on a daily basis have both desirable and undesirable outcomes and these choices may be later regretted by the person who made the choice or may be socially undesirable. Identification of variables that influence choice will allow individuals, clinicians, and policy makers to engineer choice situations that change behavioral outcomes. It is well established that the delay and intensity of the delayed aversive event influence choice, but there is less known about how signaled aversive events might influence choice (Meindl \& Casey, 2012). An understanding of the role of signals would be valuable given that the presence or absence of a signal can be relatively easily controlled.

Pitting and Scherbaum (2020) recently evaluated how consequences with both rewarding and aversive stimuli affect choice with individuals with high or low levels of anxiety. Pitting and Scherbaum modeled decision making in such situations. Participants were given a choice between a single-valence outcome consisting of 25 hypothetical cents and a dual-valence outcome consisting of larger amount of hypothetical money, the amount of which varied across conditions, and some likelihood of real electric stimulation, the probability of which varied across conditions. Choice for the dual-valence outcome decreased as the probability of the delivery of the electric stimulation was raised and choice for the dual-valence outcome increased as the amount of reward was raised. Individuals with high anxiety tended to choose the singlevalence option more relative to low anxiety individuals when the probability of the aversive stimulus was higher. The elevated avoidance of the potential aversive outcome can be 
problematic for individuals with anxiety because avoidance can also result in loss of rewarding outcomes (Craske et al., 2017). Research on methods to reduce the aversiveness of these events, such as by signaling them, may inform treatment for individuals with anxiety. Parameters of and conditions under which such signals are most effective can and should be studied in the basic laboratory to inform social engineers of the most potentially useful methods to reduce undesirable behavior. 


\section{References}

Badia, P., Coker, C., \& Harsh, J. (1973). Choice of higher density signalled shock over lower density unsignalled shock. Journal of the Experimental Analysis of Behavior, 20(1), 4755. https://doi.org/10.1901/jeab.1973.20-47

Badia, P., \& Culbertson, S. (1972). The relative aversiveness of signalled vs unsignalled escapable and inescapable shock. Journal of the Experimental Analysis of Behavior, 17(3), 463-471. https://doi.org/10.1901/jeab.1972.17-463

Badia, P., Culbertson, S., \& Harsh, J. (1973). Choice of longer or stronger signalled shock over shorter or weaker unsignalled shock. Journal of the Experimental Analysis of Behavior, 19(1), 25-32. https://doi.org/10.1901/jeab.1973.19-25

Badia, P., Harsh, J., \& Abbott, B. (1979). Choosing between predictable and unpredictable shock conditions: Data and Theory. Psychological Bulletin, 86(5), 1107-1131. https://doi.org/10.1037/0033-2909.86.5.1107

Craske, M. G., Stein, M. B., Eley, T. C., Milad, M. R., Holmes, A., Rapee, R. M., et al. (2017). Anxiety disorders. Nature Reviews Disease Primers, 3, 17024. https://doi.org/10.1038/nrdp.2017.24

Davis, H., \& McIntire, R. W. (1969) Conditioned suppression under positive, negative, and no contingency between conditioned and unconditioned stimuli. Journal of the Experimental Analysis of Behavior, 12, 633-640. https://doi.org/10.1901/jeab.1969.12-633

Dumas, A. D. (2014). Choice between reinforcers with and without delayed shock. Unpublished master's thesis, West Virginia University, Morgantown, West Virginia.

Harsh, J., \& Badia, P. (1975) Choice for signaled over unsignaled shock as a function of shock intensity. Journal of the Experimental Analysis of Behavior, 23, 349-355. https://doi.org/10.1901/jeab.1975.23-349 
Harsh, J., \& Badia, P. (1976) A temporal parameter influencing choice between signaled and unsignaled shock schedules. Journal of the Experimental Analysis of Behavior, 25, 327333. https://doi.org/10.1901/jeab.1976.25-327

Hymowitz, N. (1973a). Comparisons between variable-interval and fixed-interval schedules of electric shock delivery. Journal of the Experimental Analysis of Behavior, 1973, 19, 101111. https://doi.org/10.1901/jeab.1973.19-101

Hymowitz, N. (1973b). Effects of the lever-press-dependent and independent electric shock on schedule-induced water intake. The Psychological Record, 23, 487-497. https://doi.org/10.1007/BF03394196

Hymowitz, N. (1979). Suppression of responding during signaled and unsignaled shock. Psychological Bulletin, 86(1), 175-190. https://doi.org/10.1037/0033-2909.86.1.175

Imada, H. (1972). Emotional reactivity and conditionability in four strains of rats. Journal of Comparative and Physiological Psychology, 79(3), 474-480. https://doi.org/10.1037/h0032834

Liley, A. E., Gabriel, D. B. K., Sable, H. J., \& Simon, N. W. (2019). Sex differences and effects of predictive cues on delayed punishment discounting. Cognition and Behavior, 6(4), https://doi.org/10.1523/ENEURO.0225-19.2019

MacDonald, L. (1973). The relative aversiveness of signalled versus unsignaled shockpunishment. Journal of the Experimental Analysis of Behavior, 20, 37-46. https://doi.org/10.1901/jeab.1973.20-37

MacDonald, L, \& Baron, A. (1973). A rate measure of the relative aversiveness of signaled vs unsignaled shock. Journal of the Experimental Analysis of Behavior, 19, 33-38. https://doi.org/10.1901/jeab.1973.19-33 
Madden, G. J., \& Bickel, W. K. (2010). Impulsivity: The behavioral and neurological science of discounting. Washington: American Psychological Association.

Mazur, J. E. (1987). An adjusting procedure for studying delayed reinforcement. In M. L. Commons, J. E. Mazur, J. A. Nevin, \& H. Rachlin (Eds.), Quantitative analyses of behavior: Vol 5. The effect of delay and intervening events on reinforcement value (pp. 55-73). Hillsdale, NJ: Erlbaum.

McAllister, W. R., \& McAllister, D. E. (1971). Behavioral measurement of conditioned fear. In F. R. Brush (Ed.), Aversive conditioning and learning. New York: Academic Press, 1971. https://doi.org/10.1016/B978-0-12-137950-6.50007-9

Meindl, J.N., \& Casey, L.B. (2012). Increasing the suppressive effect of delayed punishers: A review of basic and applied literature. Behavioral Interventions, 27(3), 129-150. https://doi.org/10.1002/bin.1341

Miyashita, T. (1971) Proper control procedure in the "on-the-base-line" type of conditioned emotional response experiment. Japanese Psychological Research, 13, 176-182.

Nageishi, V., \& Imada, H. (1974). Suppression of licking behavior in rats as a function of predictability of shock and probability of conditioned-stimulus-shock pairings. Journal of Comparative and Physiological Psychology, 87, 1165-1173. https://doi.org/10.1037/h0037597

Perkins, C. C., Seyman, R. G., Levis, D. J., \& Spencer, H. R. (1966). Factors affecting preferences of signal-shock over shock-signal. Journal of Experimental Psychology, 72, 190-196. https://doi.org/10.1037/h0023491

Pierce, W. D., \& Cheney, C. D. (2013). Behavior analysis and learning (5th ed.). Psychology Press. https://doi.org/10.4324/9780203441817 
Pitting, A., \& Scherbaum, S. (2020). Costly avoidance in anxious individuals: Elevated threat avoidance in anxious individuals under high, but no low competing rewards. Journal of Behavior Therapy and Experimental Psychiatry, 66, 101524.

https://doi.org/10.1016/j.jbtep.2019.101524

Price, K. P., \& Geer, J.H. (1972). Predictable and unpredictable aversive events: Evidence for the safety-signal hypothesis. Psychonomic Science, 26, 215-216. https://doi.org/10.3758/BF03328598

Rodriguez, W., Bouzas, A., \& Orduña, V. (2017). Temporal discounting of aversive consequences in rats. Learning \& Behavior, 46(1), 38-48. https://doi.org/10.3758/s13420017-0279-9

Seligman, M. E. P. (1968). Chronic fear produced by unpredictable electric shock. Journal of Comparative and Physiological Psychology, 66, 402-411. https://doi.org/10.1037/h0026355

Skinner, B. F. (1953). Science and human behavior. New York: Macmillan.

Toegel, F.J. (2018). Effects of delay and signals on choice between delayed food and immediate food with delayed shock. Unpublished dissertation, West Virginia University, Morgantown, West Virginia

Trenholme, I. A., \& Baron, A. (1975). Immediate and delayed punishment of human behavior by loss of reinforcement. Learning and Motivation, 6, 62-79. https://doi.org/10.1016/0023$9690(75) 90035-1$

Weiss, K. M., \& Strongman, K. T. (1969). Shock-induced response bursts and suppression. Psychonomic Science, 15, 238-240. https://doi.org/10.3758/BF03337399 
Woolverton, W. L., Freeman, K. B., Myerson, J., \& Green, L. (2012). Suppression of cocaine self-administration in monkeys: Effects of delayed punishment. Psychopharmacology, 220(3), 509-517. https://doi.org/10.1007/s00213-011-2501-3

Woolverton W. L. (2003). A novel choice method for studying drugs as punishers.

Pharmacology, Biochemistry, and Behavior, 76(1), 125-131.

https://doi.org/10.1016/s0091-3057(03)00219-3 


\section{Table 1}

Experimental conditions for each rat (SR1, SR2, SR25, SR4) and the number of sessions in each. The conditions are defined in terms of the intensity of the shock (mA) and the presence or absence of a signal preceding shock. Conditions are listed in order of presentation. Except in the last two conditions of Rat SR2, shocks lasted $200 \mathrm{~ms}$.

\begin{tabular}{|c|c|c|c|c|c|c|c|c|c|c|c|}
\hline \multicolumn{3}{|c|}{ SR1 } & \multicolumn{3}{|c|}{ SR2 } & \multicolumn{3}{|c|}{ SR25 } & \multicolumn{3}{|c|}{ SR4 } \\
\hline $\mathrm{mA}$ & Signal & Sessions & $\mathrm{mA}$ & Signal & Sessions & $\mathrm{mA}$ & Signal & Sessions & $\mathrm{mA}$ & Signal & Sessions \\
\hline 0.4 & - & 9 & 0.4 & - & 7 & 0.4 & Yes & 8 & 0.4 & Yes & 8 \\
\hline 0.6 & - & 8 & 0.6 & - & 9 & 0.6 & Yes & 12 & 0.6 & Yes & 9 \\
\hline 0.8 & - & 25 & 0.8 & - & 34 & 0.8 & Yes & 38 & 0.8 & Yes & 58 \\
\hline 1.0 & - & 32 & 1.0 & - & 11 & 1.0 & Yes & 13 & 1.0 & Yes & 14 \\
\hline 1.0 & Yes & 10 & 0.8 & - & 18 & 1.0 & - & 71 & 1.0 & - & 34 \\
\hline 0.8 & Yes & 11 & 0.8 & Yes & 16 & 0.8 & - & 23 & 0.8 & - & 23 \\
\hline 0.9 & Yes & 26 & 1.0 & Yes & 10 & 1.0 & - & 54 & 0.6 & - & 26 \\
\hline 0.9 & - & 13 & 1.0 & - & 14 & & & & 1.0 & Yes & 12 \\
\hline 0.9 & Yes & 10 & 0.8 & - & 14 & & & & 0.8 & Yes & 33 \\
\hline \multirow[t]{2}{*}{0.8} & Yes & 11 & $0.8^{\mathrm{a}}$ & - & 22 & & & & 0.6 & Yes & 15 \\
\hline & & & $0.8^{\mathrm{b}}$ & - & 15 & & & & & & \\
\hline
\end{tabular}

${ }^{\text {a }}$ Shock duration was $300 \mathrm{~ms}$.

${ }^{\mathrm{b}}$ Shock duration was $500 \mathrm{~ms}$. 
Table 2a

No-Signal Conditions: Stable adjusting delays (s), forced-choice latencies $(\mathrm{s})\left(25^{\text {th }}, 50^{\text {th }}, 75^{\text {th }}\right.$ percentiles) and results of sign tests based on the 96 stable blocks of each condition. A '+' was assigned when a dual-valence latency was shorter than a single-valence latency, and a '-' was assigned when a dual-valence latency was longer than a single-valence latency. A difference of 0 was not included in the analysis. The right most column shows the p-values that correspond with the sign tests. Missing p-values indicate that the results were not statistically significant.

\begin{tabular}{|c|c|c|c|c|c|c|c|c|c|c|c|}
\hline \multirow[b]{2}{*}{ Rat } & \multirow[b]{2}{*}{ Condition } & \multirow{2}{*}{$\begin{array}{l}\text { Adjusting } \\
\text { Delay }\end{array}$} & \multicolumn{3}{|c|}{ Single Valence } & \multicolumn{3}{|c|}{ Dual Valence } & \multicolumn{3}{|c|}{ Sign Test } \\
\hline & & & 25 th & 50 th & 75 th & 25 th & 50 th & 75 th & + & - & $\mathrm{p}$ \\
\hline \multirow[t]{5}{*}{ SR1 } & 0.4 & 10.00 & 0.83 & 0.87 & 1.01 & 0.79 & 1.12 & 1.47 & 39 & 54 & -- \\
\hline & 0.6 & 10.00 & 0.83 & 0.83 & 1.03 & 1.10 & 1.24 & 1.42 & 21 & 75 & $<.001$ \\
\hline & 0.8 & 41.25 & 0.81 & 0.83 & 0.93 & 0.86 & 1.24 & 1.72 & 31 & 64 & $<.001$ \\
\hline & 1.0 & 41.75 & 0.80 & 0.83 & 0.94 & 0.81 & 1.18 & 1.49 & 31 & 64 & $<.001$ \\
\hline & 0.9 & 26.08 & 0.78 & 0.80 & 0.85 & 0.66 & 0.84 & 1.17 & 46 & 48 & -- \\
\hline \multirow[t]{9}{*}{ SR2 } & 0.4 & 10.00 & 1.08 & 1.51 & 2.47 & 0.73 & 0.96 & 2.03 & 65 & 31 & $<.001$ \\
\hline & 0.6 & 10.00 & 0.86 & 1.05 & 1.30 & 0.73 & 0.94 & 1.24 & 59 & 37 & .015 \\
\hline & 0.8 & 99.79 & 0.78 & 0.98 & 1.26 & 0.84 & 1.22 & 1.54 & 35 & 61 & .005 \\
\hline & 1.0 & 27.64 & 0.78 & 0.98 & 1.20 & 0.82 & 1.03 & 2.09 & 42 & 54 & -- \\
\hline & 0.8 & 30.21 & 0.76 & 0.98 & 1.42 & 0.67 & 0.92 & 1.57 & 52 & 42 & -- \\
\hline & 1.0 & 10.10 & 1.27 & 1.51 & 1.81 & 0.86 & 1.04 & 1.26 & 82 & 13 & $<.001$ \\
\hline & 0.8 & 10.10 & 1.27 & 1.47 & 1.82 & 0.78 & 0.91 & 1.06 & 88 & 7 & $<.001$ \\
\hline & $0.8^{\mathrm{a}}$ & 10.10 & 1.13 & 1.39 & 1.59 & 0.73 & 0.86 & 1.08 & 82 & 14 & $<.001$ \\
\hline & $0.8^{\mathrm{b}}$ & 12.43 & 1.08 & 1.31 & 1.51 & 0.75 & 1.02 & 1.35 & 65 & 31 & $<.001$ \\
\hline \multirow[t]{3}{*}{ SR25 } & $1.0^{\mathrm{c}}$ & 54.99 & 1.06 & 1.48 & 2.09 & 1.06 & 1.33 & 1.83 & 269 & 209 & -- \\
\hline & 0.8 & 14.21 & 0.80 & 1.05 & 1.40 & 0.94 & 1.12 & 1.28 & 39 & 57 & .041 \\
\hline & 1.0 & 59.10 & 1.40 & 2.00 & 2.64 & 1.01 & 1.29 & 1.57 & 78 & 18 & $<.001$ \\
\hline \multirow[t]{3}{*}{ SR4 } & 1.0 & 49.75 & 0.76 & 0.86 & 1.05 & 0.75 & 0.94 & 1.35 & 38 & 58 & .026 \\
\hline & 0.8 & 55.02 & 0.75 & 0.94 & 1.26 & 0.71 & 1.04 & 1.37 & 47 & 49 & -- \\
\hline & 0.6 & 59.48 & 1.07 & 1.44 & 2.06 & 0.94 & 1.23 & 1.47 & 60 & 35 & .006 \\
\hline
\end{tabular}

a Shock duration was $300 \mathrm{~ms}$.

${ }^{\mathrm{b}}$ Shock duration was $500 \mathrm{~ms}$.

${ }^{\mathrm{c}}$ Sign test was calculated from the 480 blocks used to calculate the adjusting delay. 
Table 2b

Signal Conditions: Stable adjusting delays (s), forced-choice latencies (s) $\left(25^{\text {th }}, 50^{\text {th }}, 75^{\text {th }}\right.$ percentiles) and results of sign tests. Details as in Table $2 a$.

\begin{tabular}{|c|c|c|c|c|c|c|c|c|c|c|c|}
\hline \multirow[b]{2}{*}{ Rat } & \multirow[b]{2}{*}{ Condition } & \multirow{2}{*}{$\begin{array}{c}\text { Adjusting } \\
\text { Delay }\end{array}$} & \multicolumn{3}{|c|}{ Single Valence } & \multicolumn{3}{|c|}{ Dual Valence } & \multicolumn{3}{|c|}{ Sign Test } \\
\hline & & & 25th & 50 th & 75 th & 25th & 50 th & 75 th & + & - & $\mathrm{p}$ \\
\hline \multirow[t]{5}{*}{ SR1 } & 1.0 & 44.19 & 0.80 & 0.81 & 0.90 & 0.66 & 0.80 & 1.31 & 48 & 47 & -- \\
\hline & 0.8 & 13.10 & 0.78 & 0.80 & 0.87 & 0.61 & 0.69 & 0.81 & 72 & 24 & $<.001$ \\
\hline & 0.9 & 37.25 & 0.80 & 0.80 & 0.82 & 0.64 & 0.94 & 1.22 & 41 & 55 & -- \\
\hline & 0.9 & 26.08 & 0.80 & 0.81 & 0.84 & 0.66 & 0.91 & 1.26 & 40 & 55 & -- \\
\hline & 0.8 & 61.15 & 0.78 & 0.80 & 0.84 & 0.66 & 0.95 & 1.20 & 34 & 61 & .003 \\
\hline \multirow[t]{2}{*}{$\mathrm{SR} 2$} & 0.8 & 12.77 & 1.26 & 1.50 & 1.96 & 0.82 & 1.03 & 1.42 & 75 & 21 & $<.001$ \\
\hline & 1.0 & 10.50 & 1.06 & 1.49 & 1.79 & 0.82 & 1.10 & 1.40 & 70 & 26 & $<.001$ \\
\hline \multirow[t]{4}{*}{ SR25 } & 0.4 & 10.30 & 0.69 & 1.12 & 1.38 & 0.59 & 0.64 & 0.74 & 72 & 23 & $<.001$ \\
\hline & 0.6 & 10.50 & 0.67 & 0.94 & 1.16 & 0.66 & 0.69 & 0.78 & 63 & 32 & $<.001$ \\
\hline & 0.8 & 12.56 & 0.83 & 0.97 & 1.24 & 0.56 & 0.61 & 0.67 & 87 & 9 & $<.001$ \\
\hline & 1.0 & 10.08 & 0.97 & 1.15 & 1.42 & 0.62 & 0.67 & 0.73 & 91 & 4 & $<.001$ \\
\hline \multirow[t]{7}{*}{ SR4 } & 0.4 & 10.00 & 0.84 & 1.09 & 1.46 & 0.62 & 0.75 & 0.91 & 76 & 19 & $<.001$ \\
\hline & 0.6 & 10.00 & 0.99 & 1.26 & 1.94 & 0.56 & 0.59 & 0.72 & 90 & 6 & $<.001$ \\
\hline & 0.8 & 114.29 & 1.01 & 1.15 & 1.34 & 0.84 & 1.51 & 2.42 & 39 & 56 & -- \\
\hline & 1.0 & 126.94 & 0.92 & 1.02 & 1.19 & 0.81 & 1.33 & 2.47 & 38 & 57 & .032 \\
\hline & 1.0 & 62.69 & 0.83 & 0.94 & 1.19 & 0.75 & 0.94 & 1.28 & 57 & 39 & .041 \\
\hline & 0.8 & 115.46 & 0.87 & 1.08 & 1.39 & 0.78 & 1.07 & 1.26 & 51 & 44 & -- \\
\hline & 0.6 & 61.17 & 0.97 & 1.25 & 1.56 & 0.67 & 0.76 & 1.12 & 78 & 18 & $<.001$ \\
\hline
\end{tabular}




\section{Table 3}

The number of Signal and No-Signal conditions in which the sign test results showed that significantly less or more than 50 percent of shorter latencies occurred in the dual-valence trial, or with no significant difference. Also shown is the proportion of conditions in which a greater number of shorter latencies occurred in the dual-valence trial.

\begin{tabular}{|c|c|c|c|c|c|c|c|c|}
\hline \multirow[b]{2}{*}{ Rat } & \multicolumn{4}{|c|}{ No Signal } & \multicolumn{4}{|c|}{ Signal } \\
\hline & Below 50\% & Above $50 \%$ & $\begin{array}{c}\text { No } \\
\text { Significant } \\
\text { Difference }\end{array}$ & $\begin{array}{c}\text { \% Above } \\
50 \%\end{array}$ & Below 50\% & Above $50 \%$ & $\begin{array}{c}\text { No } \\
\text { Significant } \\
\text { Difference }\end{array}$ & $\begin{array}{c}\text { \% Above } \\
50 \%\end{array}$ \\
\hline SR1 & 2 & 0 & 1 & 0 & 1 & 1 & 3 & 20 \\
\hline SR2 & 1 & 2 & 2 & 40 & 0 & 2 & 0 & 100 \\
\hline SR25 & 1 & 1 & 1 & 33 & 0 & 2 & 0 & 100 \\
\hline SR4 & 1 & 1 & 1 & 33 & 1 & 3 & 2 & 50 \\
\hline
\end{tabular}




\section{Figure 1}

Comparison of Adjusting Delays for Signaled and Unsignaled Conditions from Dumas (2014)

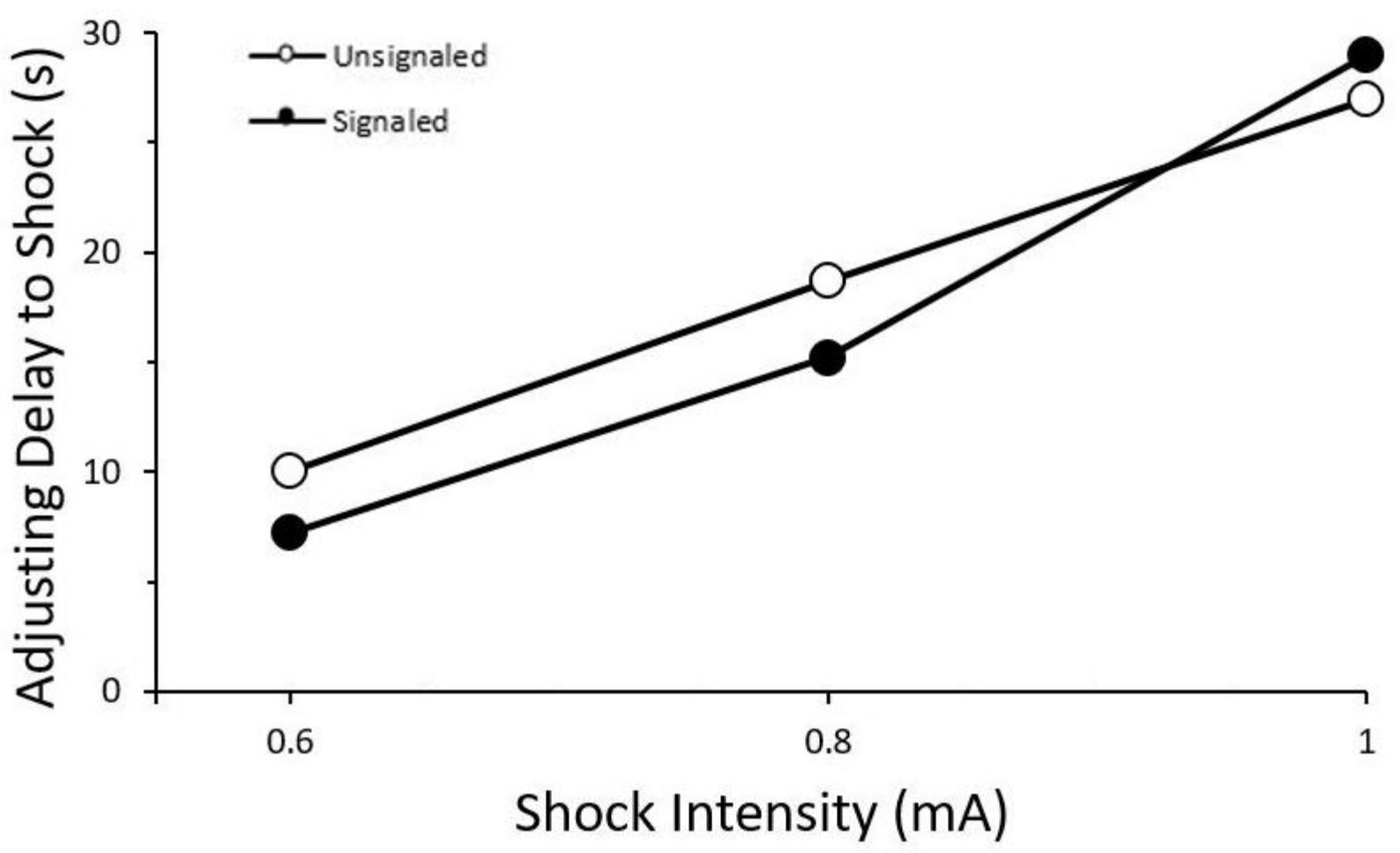

Note. Stable adjusting delays to shock for Rat AD10 at three shock intensities with and without a signal. Redrawn from Dumas (2014). 


\section{Figure 2}

Latencies to Respond as a Function of Punishment Delays from Trenholme and Baron (1975)

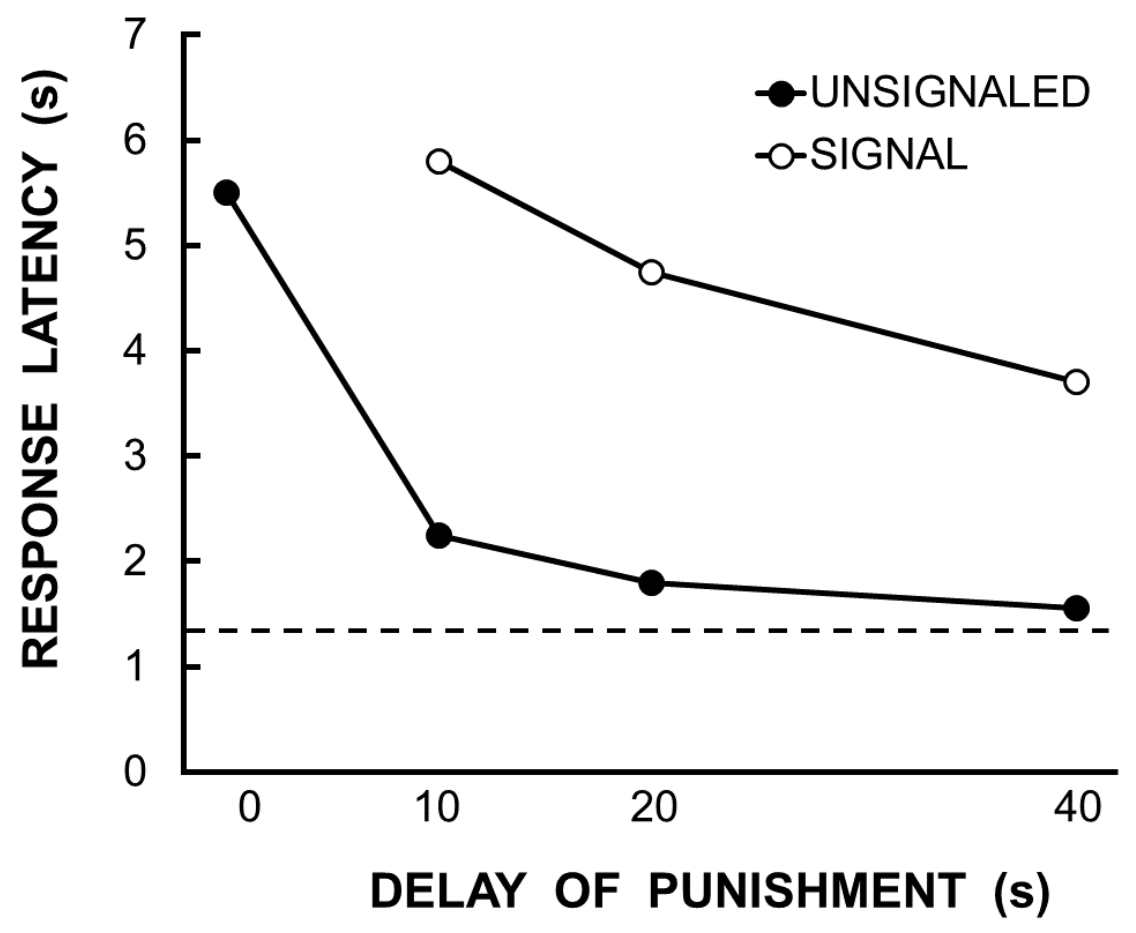

Note. Mean response latencies of groups of college students at several delays to money loss with and without a signal. The dashed line shows the mean response latency during the unpunished baseline condition. Redrawn from Trenholme and Baron (1975). 


\section{Figure 3}

Results for the 1.0 mA No-Signal Condition for Rat SR25

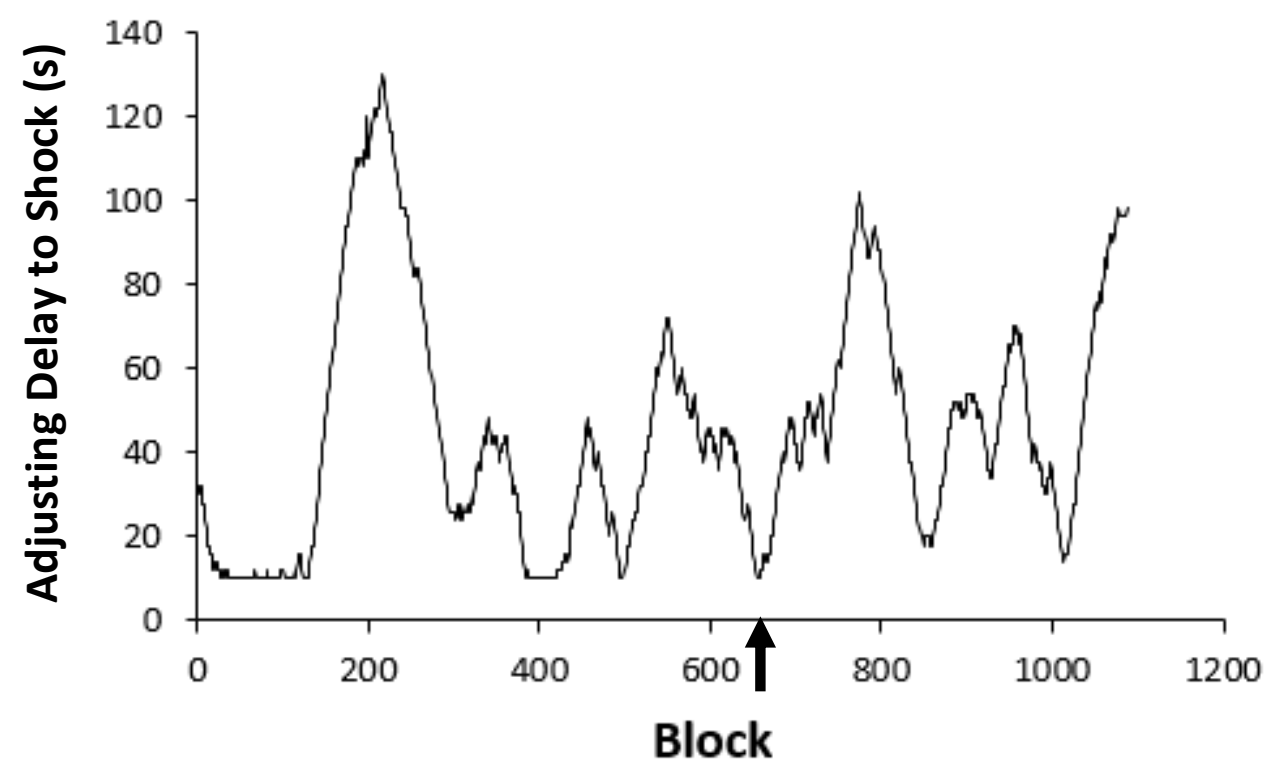

Note. Rat SR25's adjusting delay across blocks in the condition in which the last 30 sessions (480 blocks) were used to calculate the indifference point. The arrow designates the block at which stable sessions began. 


\section{Figure 4}

Stable Adjusting Delays as a Function of Shock Intensity in the Signal and No-Signal Conditions

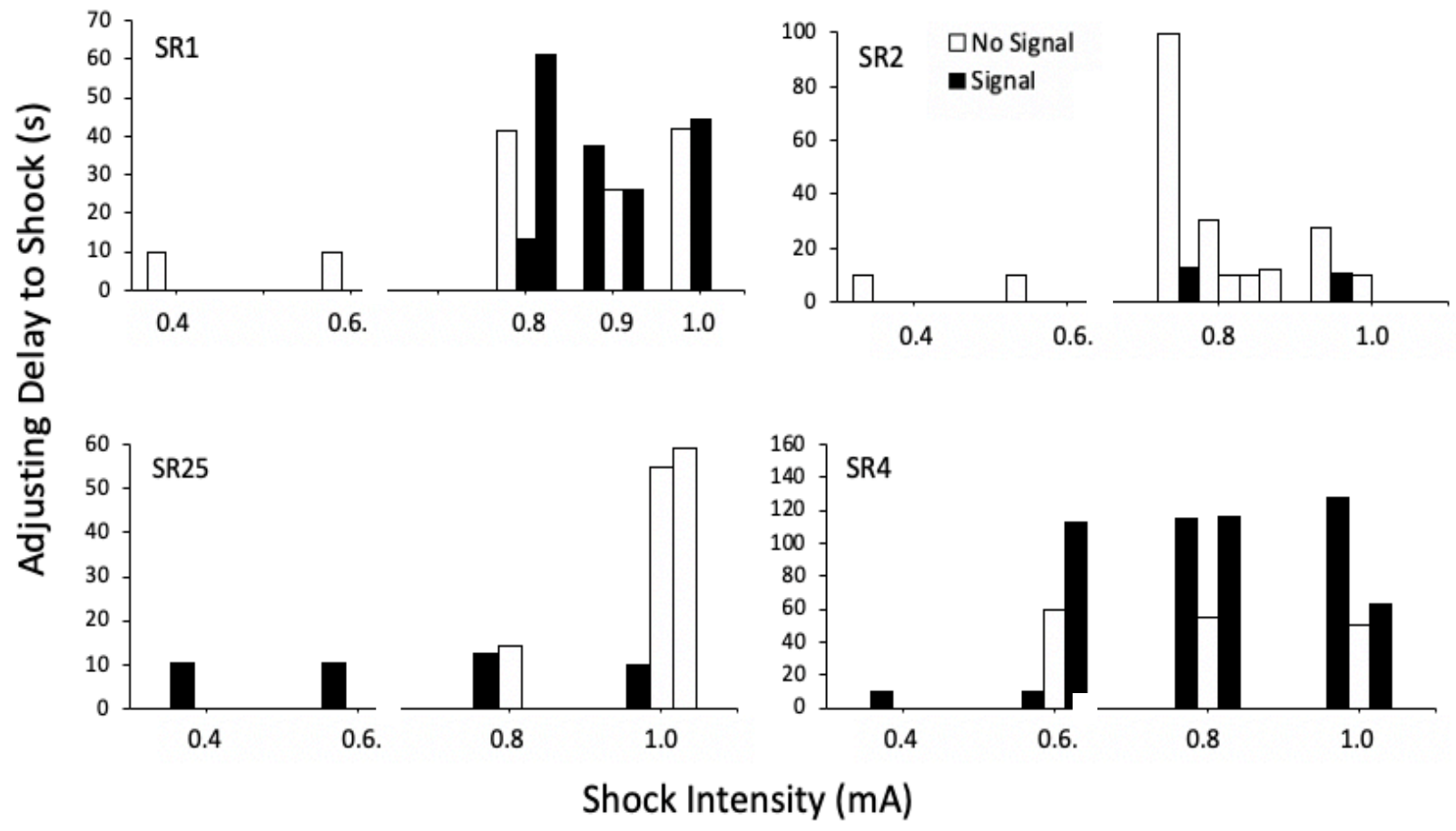

Note. Stable adjusting delays to shock from all Signal and No-Signal conditions as a function of shock intensity. Bars are displayed in order of presentation within each shock intensity. Shocks lasted $200 \mathrm{~ms}$ except in Rat SR2's last two conditions at $0.8 \mathrm{~mA}$. Here, the shocks lasted $300 \mathrm{~ms}$ and $500 \mathrm{~ms}$, respectively. Note that the scale on the y-axis differs across rats. 


\section{Figure 5}

Adjusting Delay to Shock Across Blocks for Signal and No-Signal Conditions

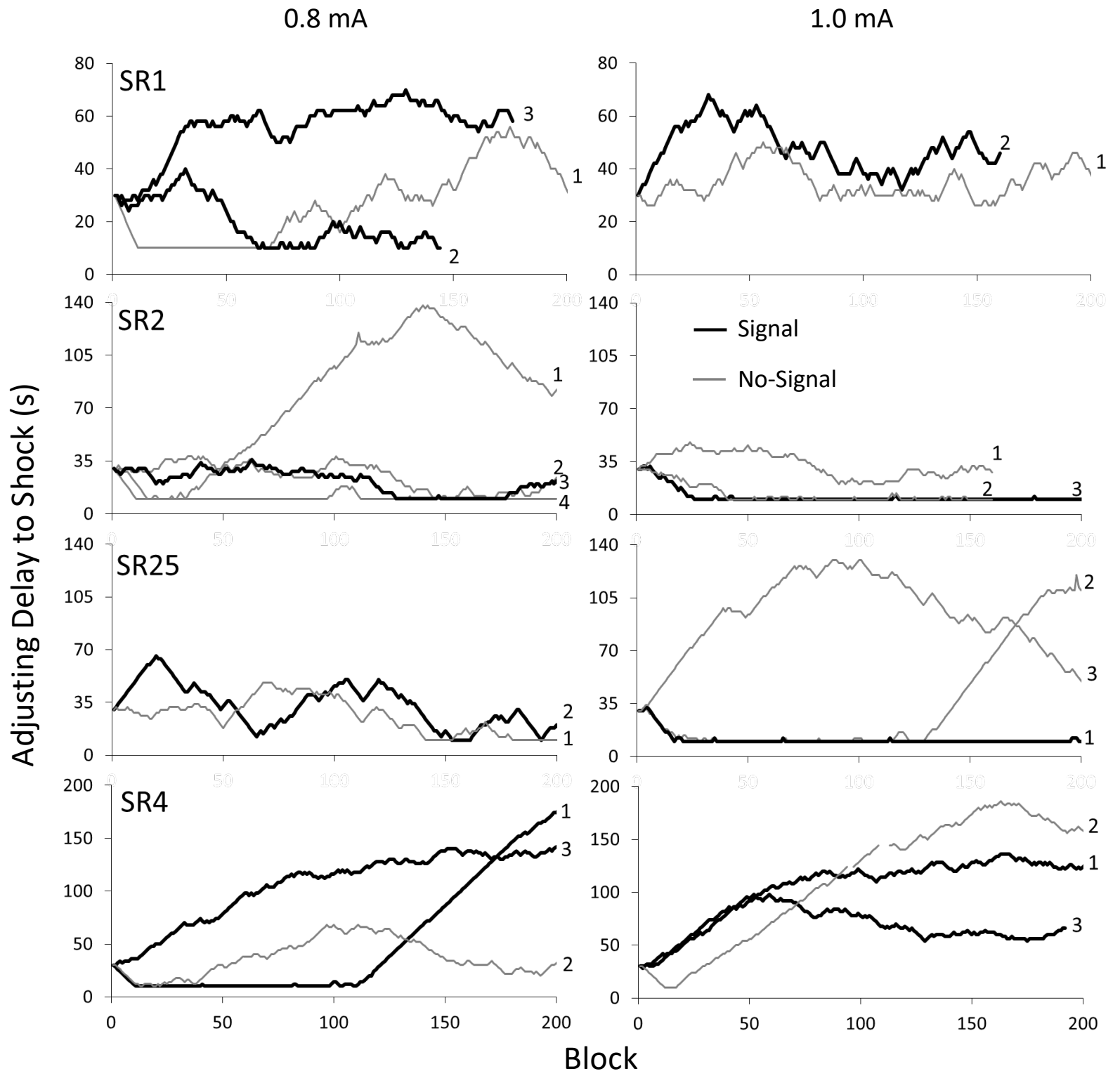

Note. Adjusting delay to shock (s) plotted across the first 200 blocks at the $0.8 \mathrm{~mA}$ and $1.0 \mathrm{~mA}$ shock intensities. Numbers next to data paths indicate the order of conditions within each shock intensity. Breaks in the second data path for Rat SR4 are a result of incomplete sessions. Note that the scale on the $y$-axis differs across rats. 


\section{Figure 6a}

Percent of Trials with Shorter Latencies for Each Lever in Forced-Choice Trials Across Shock Intensities in the No-Signal Conditions

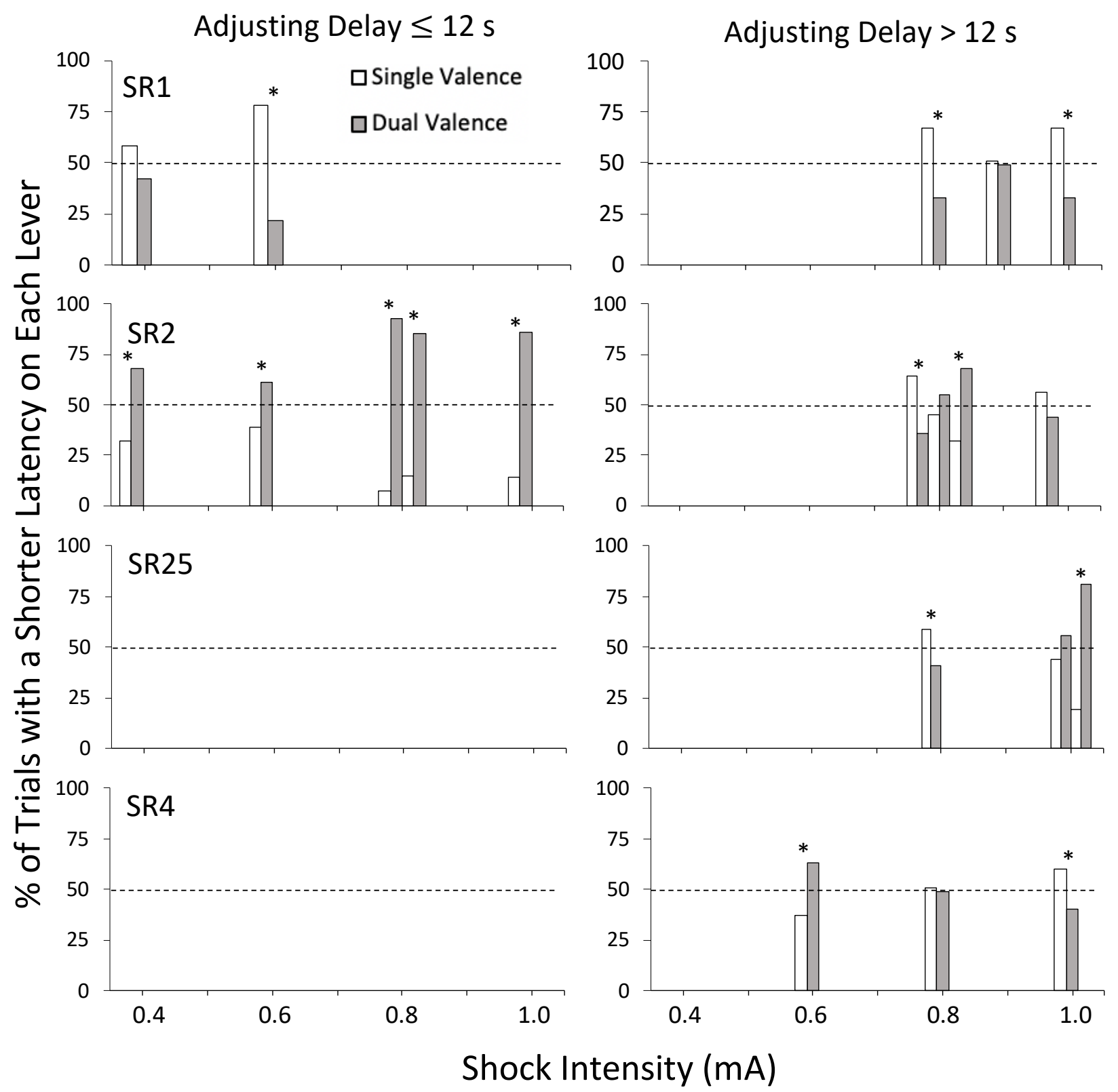

Note. Percentage of trials that the latency to respond was shorter in the dual-valence trial and in the single-valence trial during forced-choice trials in stable sessions of each No-Signal condition. The left column shows results for conditions with adjusting delays at or below $12 \mathrm{~s}$; the right column shows results for conditions with adjusting delays above $12 \mathrm{~s}$. The asterisks above pairs of bars indicate that the difference in number of longer and shorter latencies across the two levers was statistically significant. 


\section{Figure 6b}

Percentage of Shorter Latencies for Each Lever in Forced-Choice Trials Across Shock Intensities in the Signal Conditions

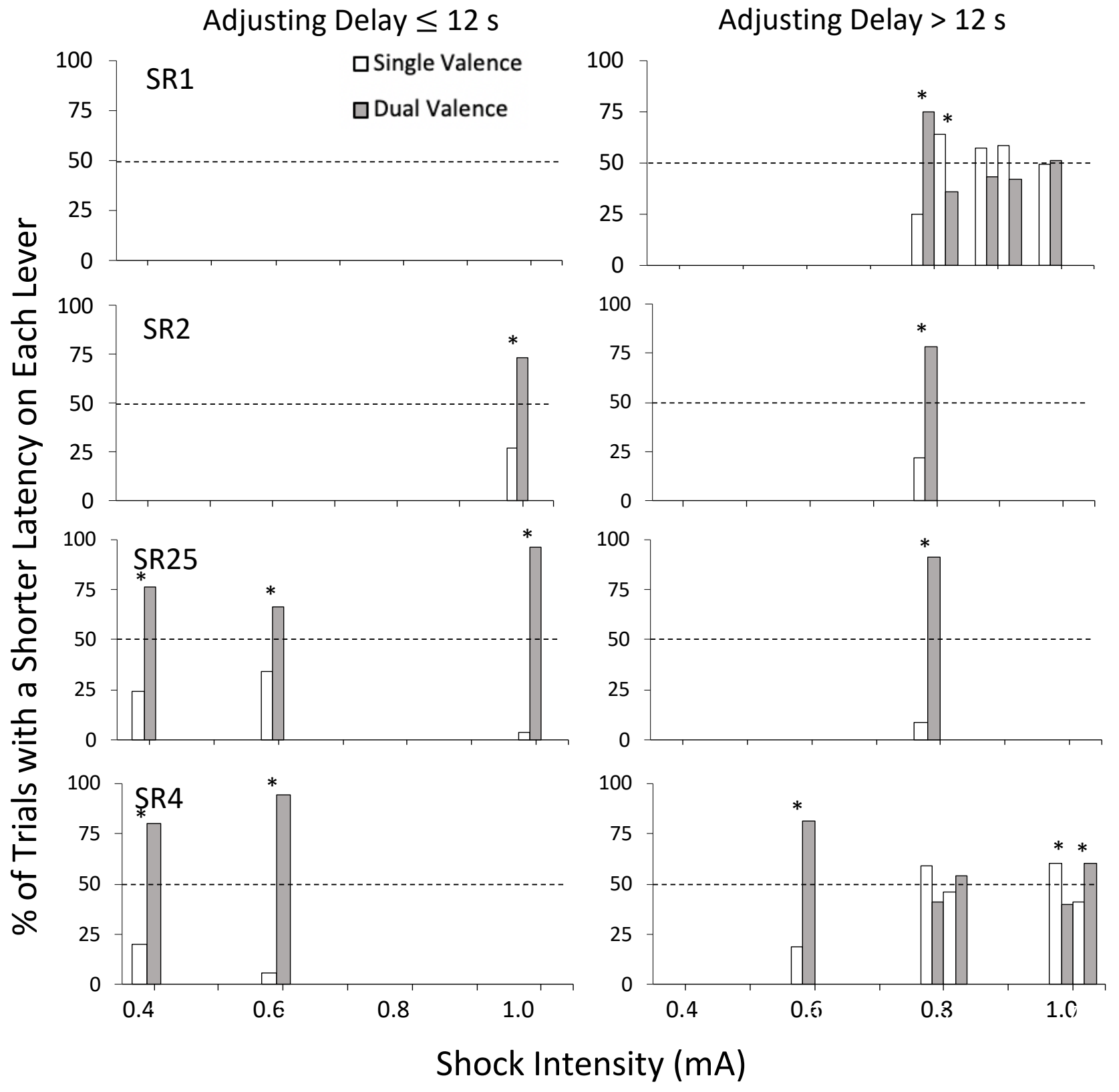

Note. Percentage of trials that the latency to respond was shorter in the dual-valence trial (shaded) and in the single-valence trial (white) during forced-choice trials in stable of each Signal condition across shock intensity. Details as in Figure 6a. 


\section{Figure 7}

Percentage of Trials with Shorter Latencies on the Dual-Valence Lever in Signal and No-Signal Conditions

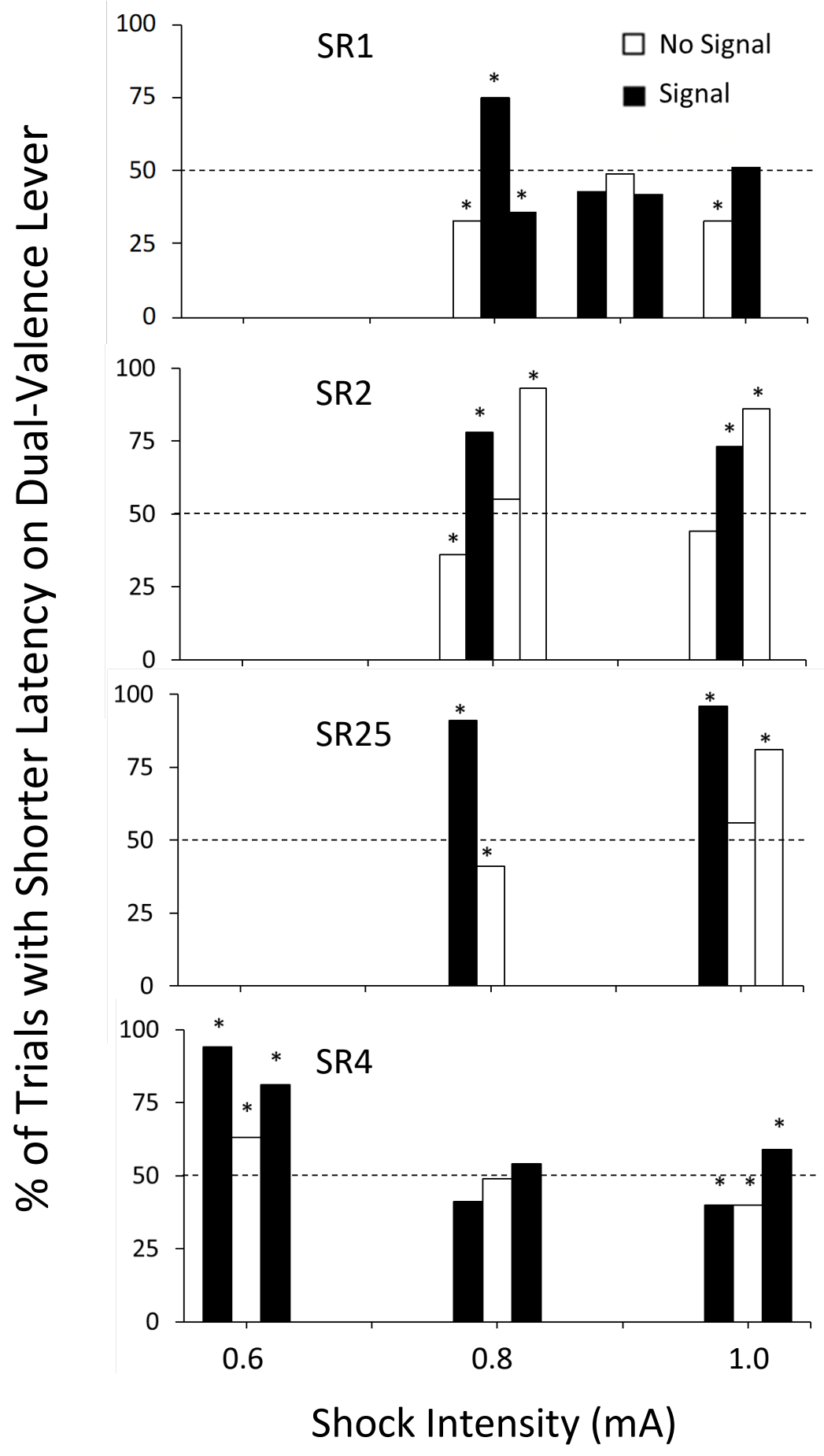

Note. Percentage of trials with a shorter latency to press the dual-valence lever during the stable sessions of Signal and No Signal conditions. 